Article

\title{
Investigating the Effect of an Oxytetracycline Treatment on the Gut Microbiome and Antimicrobial Resistance Gene Dynamics in Nile Tilapia (Oreochromis niloticus)
}

\author{
Christopher J. Payne * $\mathbb{D}$, James F. Turnbull, Simon MacKenzie and Margaret Crumlish *
}

Citation: Payne, C.J.; Turnbull, J.F.; MacKenzie, S.; Crumlish, M. Investigating the Effect of an Oxytetracycline Treatment on the Gut Microbiome and Antimicrobial Resistance Gene Dynamics in Nile Tilapia (Oreochromis niloticus). Antibiotics 2021, 10, 1213. https:// doi.org/10.3390/antibiotics10101213

\section{Academic Editors:}

David Rodríguez-Lázaro and María Ugarte Ruiz

Received: 31 August 2021

Accepted: 1 October 2021

Published: 6 October 2021

Publisher's Note: MDPI stays neutral with regard to jurisdictional claims in published maps and institutional affiliations.

Copyright: (c) 2021 by the authors. Licensee MDPI, Basel, Switzerland. This article is an open access article distributed under the terms and conditions of the Creative Commons Attribution (CC BY) license (https:// creativecommons.org/licenses/by/ $4.0 /)$.
Institute of Aquaculture, University of Stirling, Stirling FK9 4LA, UK; j.f.turnbull@stir.ac.uk (J.F.T.); simon.mackenzie@stir.ac.uk (S.M.)

* Correspondence: c.j.payne1@stir.ac.uk (C.J.P.); margaret.crumlish@stir.ac.uk (M.C.)

\begin{abstract}
Antibiotics play a vital role in aquaculture where they are commonly used to treat bacterial diseases. However, the impact of antibiotic treatment on the gut microbiome and the development of antimicrobial resistance in Nile tilapia (Oreochromis niloticus) over time remains to be fully understood. In this study, fish were fed a single treatment of oxytetracycline (100 mg/kg/day) for eight days, followed by a 14-day withdrawal period. Changes in the distal gut microbiome were measured using $16 \mathrm{~S}$ rRNA sequencing. In addition, the abundance of antimicrobial resistance genes was quantified using real-time qPCR methods. Overall, the gut microbiome community diversity and structure of Nile tilapia was resilient to oxytetracycline treatment. However, antibiotic treatment was associated with an enrichment in Plesiomonas, accompanied by a decline in other bacteria taxa. Oxytetracycline treatment increased the proportion of $t e t A$ in the distal gut of fish and tank biofilms of the treated group. Furthermore, the abundance of tet $A$ along with other tetracycline resistance genes was strongly correlated with a number of microbiome members, including Plesiomonas. The findings from this study demonstrate that antibiotic treatment can exert selective pressures on the gut microbiome of fish in favour of resistant populations, which may have long-term impacts on fish health.
\end{abstract}

Keywords: fish microbiome; tilapia; bacterial 16S rRNA gene; antibiotics; oxytetracycline; antibiotic resistance

\section{Introduction}

The aquaculture industry is a vital production sector for global food security, producing a staggering 114.5 million tonnes of aquatic protein in 2018, worth an estimated USD 263.6 billion [1] (p. 21). Within this sector, Nile tilapia (Oreochromis niloticus) is one of the most prevalent freshwater farmed fish species worldwide, contributing $8 \%$ of the global production in 2016 [2] (p. 23). Like many farmed fish species, the production of Nile tilapia is expected to intensify in the coming decades, in order to meet the growing demand for aquatic animal protein [3]. However, expansion of this sector is currently hampered by a number of challenges including infectious disease outbreaks from Gram-negative and Gram-positive pathogens [4-6], which can limit production and have huge economic impacts [7]. As a result, tilapia farmers rely on the use of antibiotics to treat or prevent bacterial diseases. Recent surveys of farmers and quantification of antibiotic residues in fish tissue have revealed the use of a diverse variety of antibiotics in tilapia farming systems from top producing countries such as Brazil, Egypt, and Vietnam [8-10]. These include antibiotics within the classes amphenicols, B-lactams, fluoroquinolones, potentiated sulfonamides, and tetracyclines including oxytetracycline (OTC).

Oxytetracycline is a natural broad-spectrum antibiotic extensively used in global aquaculture due to its limited side effects in the host and broad-spectrum activity against both Gram-negative and Gram-positive bacteria, through the inhibition of protein syn- 
thesis [11-13]. In tilapia, OTC has been shown to successfully treat a range of bacterial diseases including Francisellosis, motile Aeromonas septicemia, and Streptococcosis, which are significant disease challenges for this sector [14-16]. Across the global aquaculture industry, OTC is often administered orally in the diet during disease outbreaks, where doses range 50 to $250 \mathrm{mg} / \mathrm{kg}$ / day for typically between four and eight days, up to 21 days, depending on the fish species and farming country $[17,18]$. Within the industry, a lack of understanding in antibiotic management has led to the misuse and overuse of antibiotics including OTC in production systems, with a limited understanding of how they may impact overall fish health.

The gastrointestinal tract of Nile tilapia is colonised by a community of microorganisms or "microbiome", comprised predominantly of Fusobacteria, Firmicutes, and Proteobacteria $[19,20]$. In this fish species, gut microbiome members play a pivotal role in supporting host physiology and health, through microbial-mediated functions involved in disease resistance, growth, and metabolism [21,22]. Recent genomic studies have revealed this commensal community in Nile tilapia to be influenced by a number of factors commonly associated with aquaculture husbandry practices, such as alternations in salinity [23], dietary changes [22,24], and starvation [25]. Likewise, a number of studies have demonstrated that this commensal community in Nile tilapia and other fish species can also be altered following exposure to a number of antibiotics, including OTC $[13,17,26,27]$. However, the changes in the gut microbiome dynamics over time following OTC treatment in Nile tilapia have not been fully explored. As members within the gut microbiome serve important biological functions, understanding the short-term impacts of antibiotics on the gut microbiome is important, as any alteration or dysbiosis of this community may have detrimental effects on the physiological status of the fish host.

The continued reliance and use of antibiotics in aquaculture have contributed to the development of antimicrobial resistance (AMR) within the industry and wider environment. Indeed, high levels ( $>70 \%$ ) of resistance have been reported to one or more antibiotic compounds in bacteria recovered from fish farms in numerous countries, including China [28], India [29], Korea [30], and Switzerland [31]. Recent genomic studies have detected numerous antibiotic resistance genes (ARGs) within the gut microbiome of freshwater carp (Labeo rohita) [32] and spotted sea bass (Lateolabrax maculatus) [33], indicating that the gut microbiome may serve as a reservoir for ARGs. Furthermore, antibiotic treatment has been demonstrated to induce selective pressures on the ARGs within the gut microbiome of fish such as Atlantic salmon (Salmo salar) and pacu (Piaractus mesopotamicus), favourably supporting the development of antibiotic resistant communities [13,34], yet little is known how antibiotic treatment influences ARG dynamics in Nile tilapia.

Due to a lack of available vaccines and other efficacious prevention tools, the tilapia sector will continue to rely heavily on the use of antibiotics to treat or prevent bacterial disease outbreaks within intensive production systems. Therefore, the objective of this study was to determine the effect of a single OTC treatment on the gut microbiome and ARG abundance in Nile tilapia over time. To achieve this, high-throughput $16 \mathrm{~S}$ rRNA amplicon-sequencing and quantitative-PCR (qPCR) methods were applied to profile the changes in the microbiome community and the abundance of ARGs in the distal gut of fish before, at the end of antibiotic treatment, and throughout a two-week withdrawal period.

\section{Results}

\subsection{Fish Performance}

A statistically significant difference was not found in the final mean length, weight, and growth rate of fish between treatment groups and time $(p=0.61, p=0.80$, and $p=0.63$, respectively; Table 1). However, in general, OTC-treated fish displayed lower growth rates compared with the control fish. 
Table 1. Final mean (+SD) length and weight measurements for control or oxytetracycline (OTC)-treated Nile tilapia before, during, and after antibiotic treatment.

\begin{tabular}{cccccccc}
\hline \multirow{2}{*}{ Treatment } & \multirow{2}{*}{ Day } & \multicolumn{2}{c}{ Length $(\mathbf{c m})$} & \multicolumn{2}{c}{ Weight $(\mathrm{g})$} & \multicolumn{2}{c}{ Growth Rate $(\mathrm{g} /$ day) } \\
\cline { 3 - 7 } & & Mean & SD & Mean & SD & Mean & SD \\
\hline Baseline & 0 & 15.00 & 0.83 & 57.75 & 8.39 & 0.37 & 0.20 \\
Control & 8 & 14.63 & 0.95 & 51.88 & 8.29 & 0.33 & 0.37 \\
OTC & 8 & 14.95 & 1.41 & 54.22 & 14.12 & 0.31 & 0.25 \\
Control & 15 & 15.65 & 1.18 & 61.15 & 14.00 & 0.45 & 0.27 \\
OTC & 15 & 14.72 & 1.09 & 54.20 & 12.03 & 0.32 & 0.22 \\
Control & 22 & 15.65 & 1.04 & 62.65 & 12.90 & 0.28 & 0.18 \\
OTC & 22 & 15.17 & 0.71 & 56.37 & 8.42 & & \\
\hline
\end{tabular}

\subsection{In Vitro Antimicrobial Testing of Prepared Diets}

Zones of inhibition in bacterial growth surrounding dietary pellets were only observed on the Aeromonas hydrophila bacterial lawn exposed to the OTC-coated pellets (Figure 1). These were measured at diameters of $>25 \mathrm{~mm}$. Control pellets that lacked OTC produced no inhibition zones after $48 \mathrm{~h}$ incubation, as shown in Figure 1.
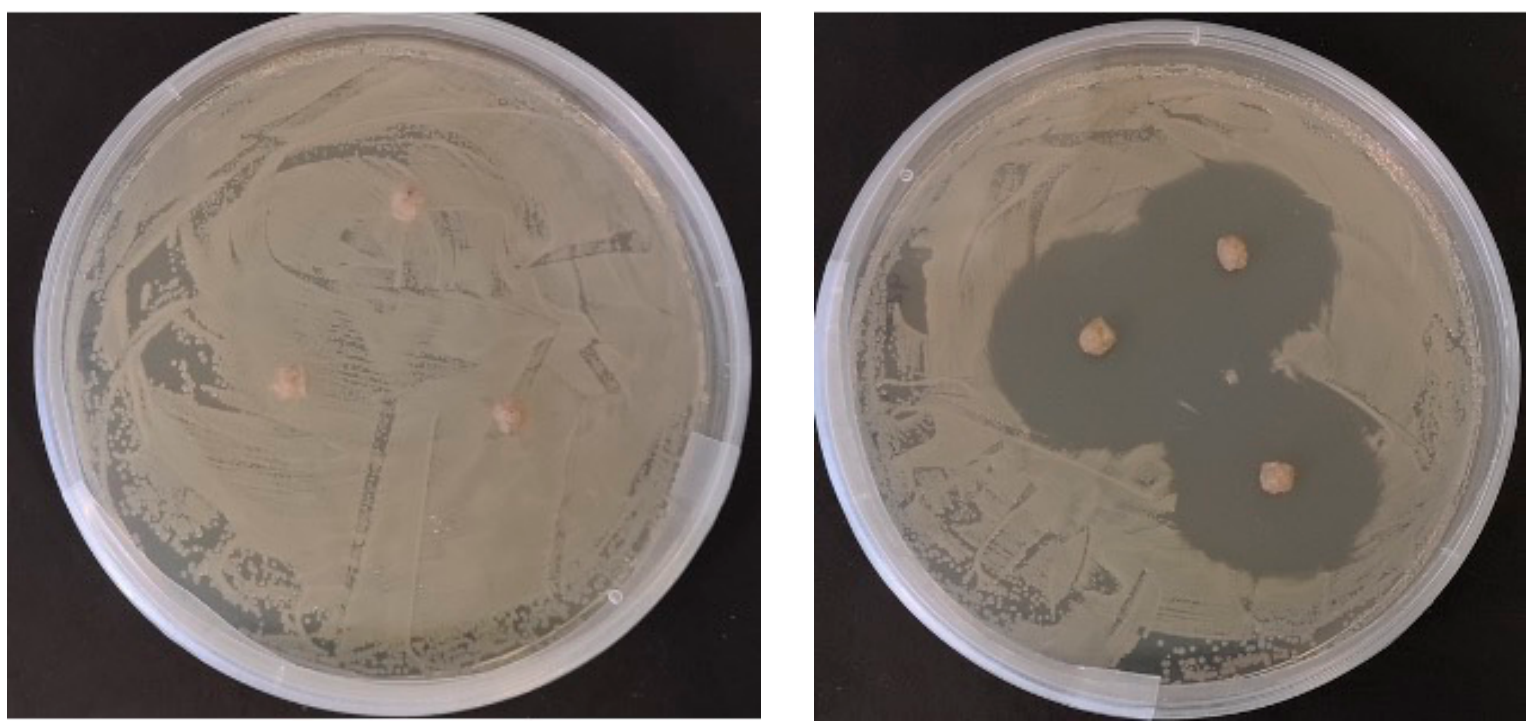

Figure 1. Antimicrobial activity of control and oxytetracycline-coated diets on Aeromonas hydrophila bacterial lawns.

\subsection{Sequence Data and Diversity Analysis}

A total of 12,733,648 reads were obtained from the Illumina MiSeq system. Following quality filtering, a total of 7,583,066 sequences remained in the final dataset and were clustered into 4450 aligned operational taxonomic units (OTUs) at a $97 \%$ similarity threshold. Of these, 2070 were observed in samples originating from fish distal gut digesta material. In addition, a total of 420,1686, 1661, and 852 OTUs were observed in either the diet, aquarium biofilter, tank biofilms, or negative sequencing control (NSC) samples, respectively. The sequencing error was calculated at $0.0105 \%$.

Data from 31 fish across treatment groups and time points were included in the bacterial analysis following sequence quality filtering and classification. Comparing the microbial alpha diversity over time found that the distal guts of fish who consumed the OTC-coated pellets had lower microbial diversity (Inverse Simpson's) and evenness (Shannon's diversity), compared with the control fish group at the end of the antibiotic treatment on day 8 (Figure 2). The mean microbial evenness decreased throughout the withdrawal period in the distal gut microbiome of the OTC-treated fish, to a level below 
that of control fish by day 22. No significant difference was observed between the treatment groups or time. Likewise, differences in the bacterial community structure were also not statistically significant, as both the microbiome community membership and composition were found to be indistinguishable by treatment group (PERMANOVA; ThetaYC: $F=0.69$, $p=0.71$; Bray-Curtis: $F=0.59, p=0.87$ ) and time (PERMANOVA; ThetaYC: $F=0.66$, $p=0.75$; Bray-Curtis: $F=1.15, p=0.30$ ) (Figure 3B,D). Distal gut microbiome communities of fish did cluster distinctly from all other sample types including NSCs (PERMANOVA; ThetaYC: $F=5.15, p<0.001$; Bray-Curtis: $F=4.74, p<0.001$ ) (Figure 3A,C). As such, the taxonomic composition of the environmental and NSC samples were not investigated further.
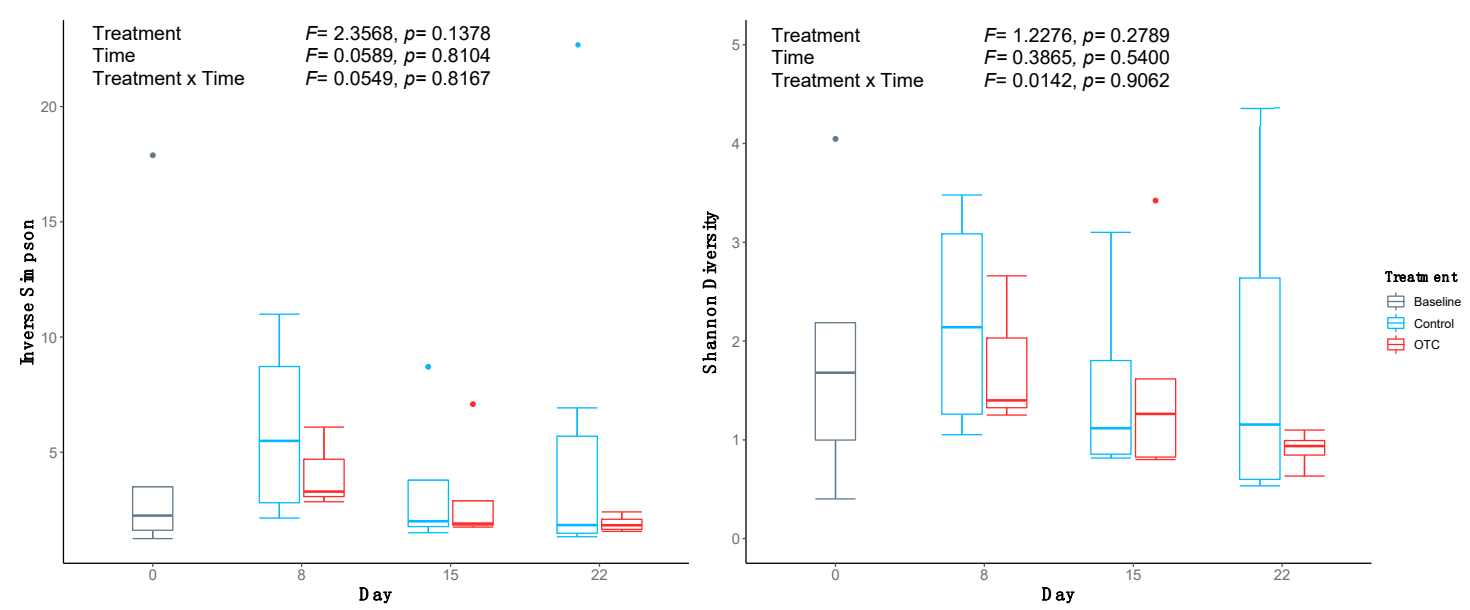

Figure 2. Alpha diversity measures of distal gut microbiome communities in control or oxytetracycline (OTC)-treated Nile tilapia before and after antibiotic treatment. Error bars indicate the $95 \%$ confidence interval; top, middle, and bottom of each box represent the 75th, 50th, and 25th percentiles, respectively. Circles indicate outliers from the dataset.
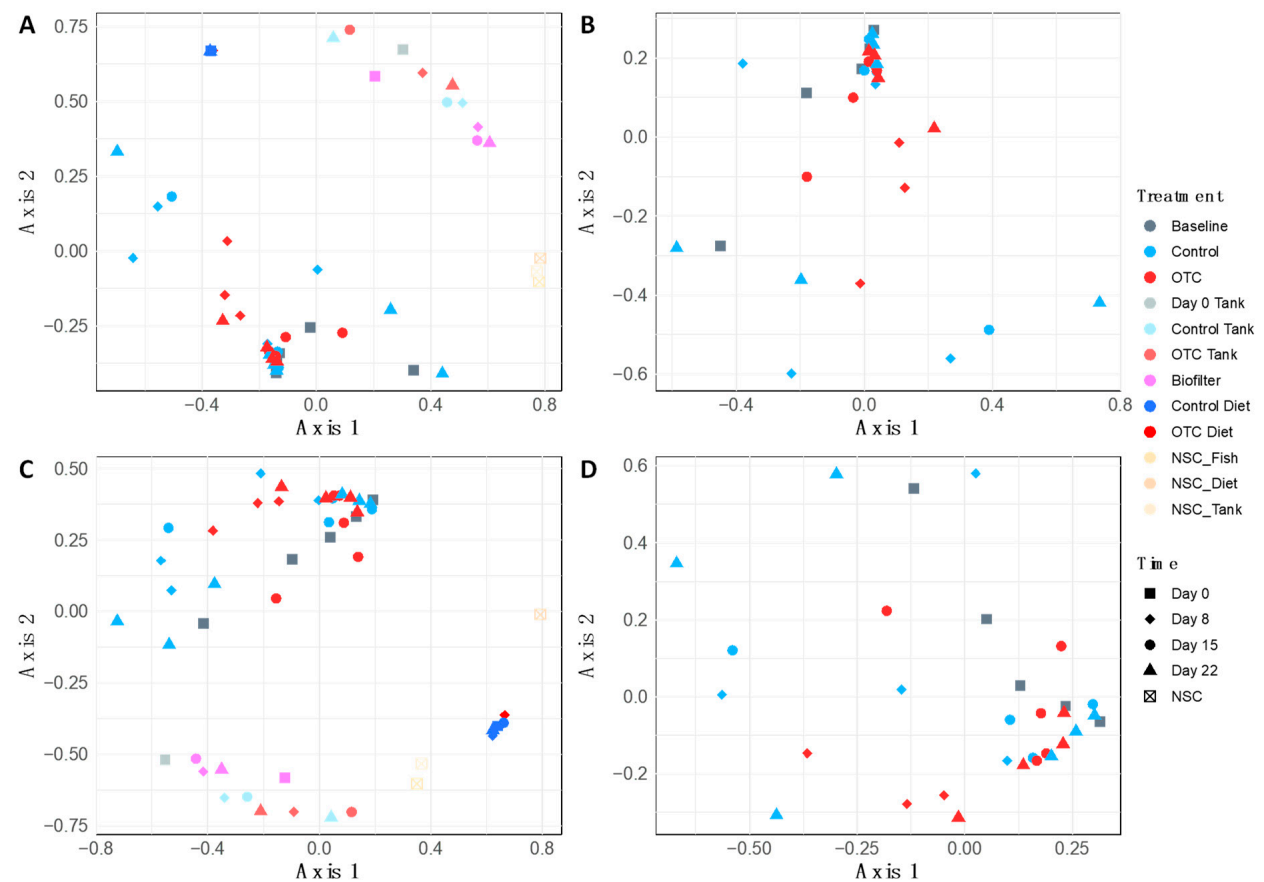

Figure 3. Non-multidimensional scaling of ThetaYC (A,B) and Bray-Curtis (C,D) distances. Distances illustrate differences in the microbiome community membership and composition of samples across time and exposure to oxytetracycline (OTC). Distances were generated for the complete dataset including aquarium biofilter, tank biofilm, diet, and negative sequencing control (NSC) samples (A,C), and within the distal gut of Nile tilapia alone (B,D). 


\subsection{Microbiome Community Dynamics in Response to OTC}

A total of 23 bacterial phyla were observed in the distal gut of Nile tilapia, with 10 being more dominant across treatment groups and time (Figure 4). The mean (+SD) abundance of the top bacterial phyla is given in Table 2. There was considerable variation found between individual fish across treatment groups and time. Figure 4 shows that the distal gut microbiome of the baseline fish on day 0 was dominated by Fusobacteria, followed by Proteobacteria, Actinobacteria, and Bacteroidetes. The mean sequence abundance of Fusobacteria was found to increase in OTC-treated fish compared with the control fish at the end of antibiotic treatment on day 8 , accompanied by a decrease in mean Proteobacteria abundance. By day 15, Actinobacteria, Chloroflexi, Firmicutes, and Planctomycetes groups were observed in higher mean abundance within the distal guts of OTC-treated fish compared with the control group. A general decline in most bacterial phyla was observed in OTC-treated fish by day 22. This shift in microbiome communities was largely driven by OTUs assigned to Fusobacteria, which was found to increase steadily in OTC-treated fish following antibiotic treatment and the withdrawal period, leading to the reduced representation of most other phyla by day 22 .

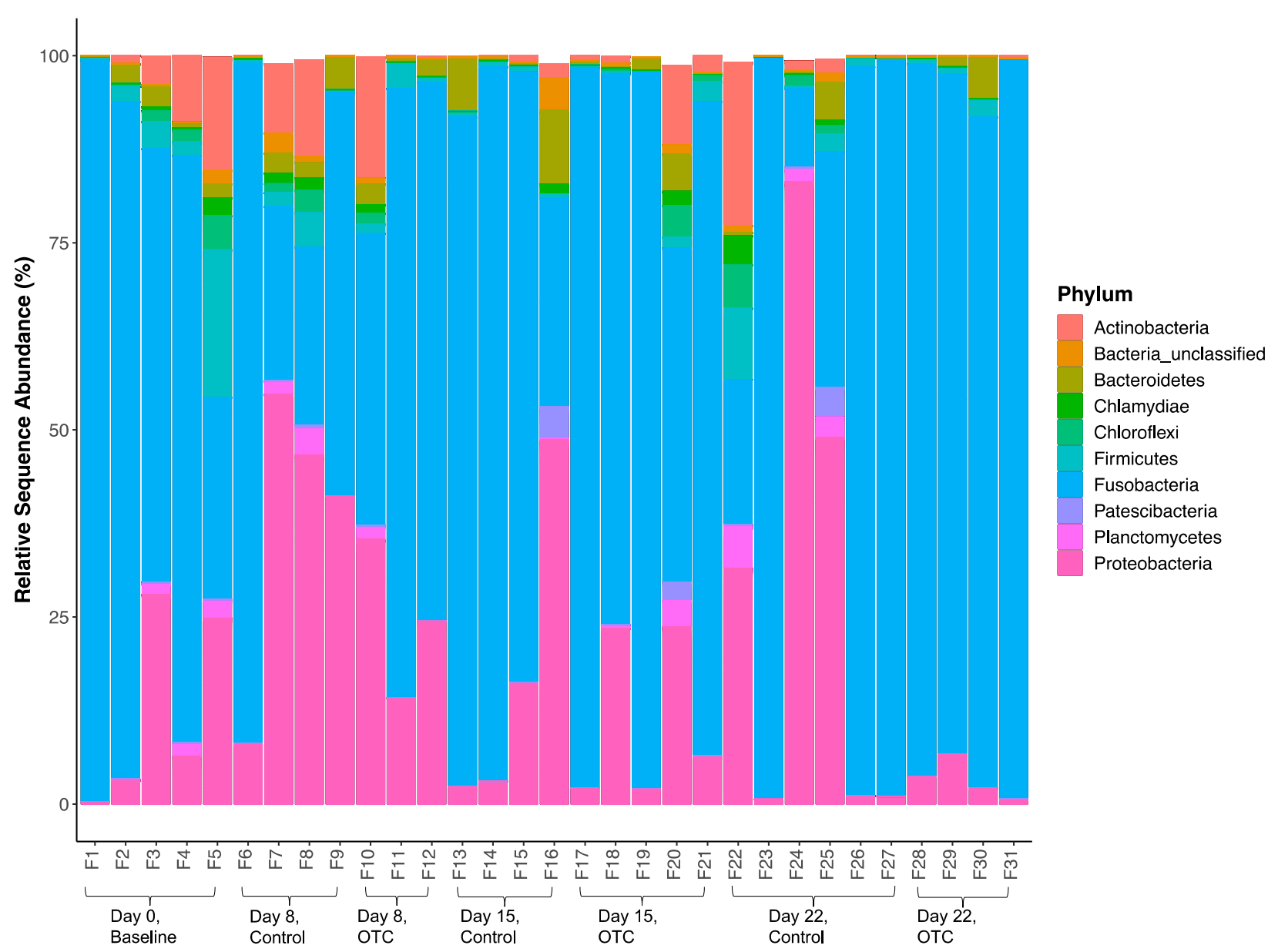

Figure 4. Relative sequence abundance (\%) of the top 10 bacterial phyla in the distal gut of control or oxytetracycline (OTC)-treated Nile tilapia before and after antibiotic treatment. 
Table 2. Mean $( \pm S D)$ abundance of top bacterial phyla in the distal gut of control or oxytetracycline (OTC)-treated Nile tilapia before and after antibiotic treatment.

\begin{tabular}{|c|c|c|c|c|c|c|c|}
\hline \multirow{2}{*}{ Phylum } & \multirow{2}{*}{$\begin{array}{c}\text { Day } 0 \\
\text { Baseline }\end{array}$} & \multicolumn{2}{|c|}{ Day 8} & \multicolumn{2}{|c|}{ Day 15} & \multicolumn{2}{|c|}{ Day 22} \\
\hline & & Control & OTC & Control & OTC & Control & OTC \\
\hline Actinobacteria & $5.78 \pm 6.24$ & $5.57 \pm 6.48$ & $5.55 \pm 9.24$ & $0.76 \pm 0.83$ & $2.89 \pm 4.39$ & $4.19 \pm 8.66$ & $0.14 \pm 0.17$ \\
\hline Bacteria_unclassified & $0.48 \pm 0.77$ & $0.87 \pm 1.24$ & $0.26 \pm 0.46$ & $1.11 \pm 2.11$ & $0.34 \pm 0.52$ & $0.40 \pm 0.53$ & $0.02 \pm 0.03$ \\
\hline Bacteroidetes & $1.58 \pm 1.24$ & $2.39 \pm 1.74$ & $1.95 \pm 1.13$ & $4.47 \pm 4.86$ & $1.51 \pm 2.07$ & $1.00 \pm 1.96$ & $1.84 \pm 2.67$ \\
\hline Chlamydiae & $0.63 \pm 0.97$ & $0.81 \pm 0.82$ & $0.38 \pm 0.66$ & $0.33 \pm 0.66$ & $0.48 \pm 0.79$ & $0.84 \pm 1.50$ & $0.01 \pm 0.01$ \\
\hline Chloroflexi & $1.62 \pm 1.76$ & $1.02 \pm 1.40$ & $0.49 \pm 0.85$ & $0.01 \pm 0.01$ & $1.06 \pm 1.80$ & $1.39 \pm 2.22$ & $0.00 \pm 0.00$ \\
\hline Firmicutes & $5.53 \pm 8.07$ & $1.83 \pm 20.02$ & $1.88 \pm 1.49$ & $0.78 \pm 0.18$ & $1.00 \pm 1.12$ & $2.26 \pm 3.69$ & $1.07 \pm 1.03$ \\
\hline Fusobacteria & $70.58 \pm 28.91$ & $47.98 \pm 32.05$ & $64.15 \pm 22.24$ & $73.58 \pm 30.96$ & $79.57 \pm 21.57$ & $59.44 \pm 43.18$ & $93.66 \pm 4.19$ \\
\hline Patescibacteria & $0.10 \pm 0.12$ & $0.16 \pm 0.20$ & $0.08 \pm 0.13$ & $1.05 \pm 2.10$ & $0.53 \pm 1.07$ & $0.73 \pm 1.58$ & $0.00 \pm 0.00$ \\
\hline Planctomycetes & $1.18 \pm 1.04$ & $1.32 \pm 1.70$ & $0.56 \pm 0.96$ & $0.06 \pm 0.09$ & $0.80 \pm 1.51$ & $1.67 \pm 2.22$ & $0.05 \pm 0.09$ \\
\hline Proteobacteria & $12.46 \pm 12.88$ & $37.59 \pm 20.50$ & $24.61 \pm 10.59$ & $17.52 \pm 21.68$ & $11.47 \pm 11.14$ & $27.70 \pm 33.76$ & $3.21 \pm 2.61$ \\
\hline
\end{tabular}

In this study, LEfSe and metastats analysis were also performed to determine which OTUs were significantly different between the OTC and control groups across time (Figure 5). Further taxonomic information is given in Table S1 (Supplementary Material). Oxytetracycline treatment was associated with a statistically significant decline in the abundance of several OTUs assigned to Actinobacteria on day 8. Several Actinobacteria OTUs could not be classified to genus level, however OTC was associated with a significant decrease in a Lamia OTU (OTU0321) on day 8. At the genus level, OTC treatment was associated with a statistically significant decline in several Proteobacteria OTUs by day 8, assigned to Aeromonas, Pseudomonas and Reyranella, amongst others. In addition, sequences from both OTU0005 (Aeromonas) and OTU0006 (Reyranella), remained depleted in the distal guts of treated fish by day 22. One Proteobacteria OTU (OTU0004), assigned to Plesiomonas, became significantly more abundant in the distal guts of treated fish on day eight compared with the control group. At day 15 , several OTUs became significantly elevated in abundance within the distal guts of treated fish. These were primarily assigned to Proteobacteria again and belonged to Aquicella (OTU0044) and Hyphomicrobium (OTU0024), amongst others. Likewise, OTUs assigned to Actinobacteria, Bacteroidetes, Chloroflexi, and Firmicutes OTUs were also found to be present at significantly higher levels, as were the Acidobacteria (OTU0065) and Chloroflexi (OTU0062) OTUs assigned to DS-100_ge and RBG-13-54-9_ge, respectively. By day 22, most differentially abundant OTUs were found to have statistically significant lower abundances in OTC-treated fish compared with control fish. These included OTUs assigned to Actinobacteria and, in particular, members belonging to Nocardioides, Mycobacterium, and a Smaragdicoccus OTU (OTU0063). In addition to Aeromonas and Reyranella OTUs, the abundance of several other Proteobacteria OTUs was also significantly lower on day 22 in OTC-treated fish compared with the control fish, including an OTU assigned to Pedomicrobium (OTU0503). Likewise, by day 22, OTUs from several other phyla also became significantly less abundant, including Chlamydiae and Verrucomicrobia, as well as several Planctomycete OTUs, assigned to Gematta (OTU0262 and OTU0588) and Planctopirus (OTU0073). This overall decline in most OTUs by day 22 was attributed to OTU0002 and OTU0020, assigned to Cetobacterium (Fusobacteria) and Macellibacteroides (Bacteroidetes), respectively, which were present at significantly higher abundances in OTC-treated fish. 


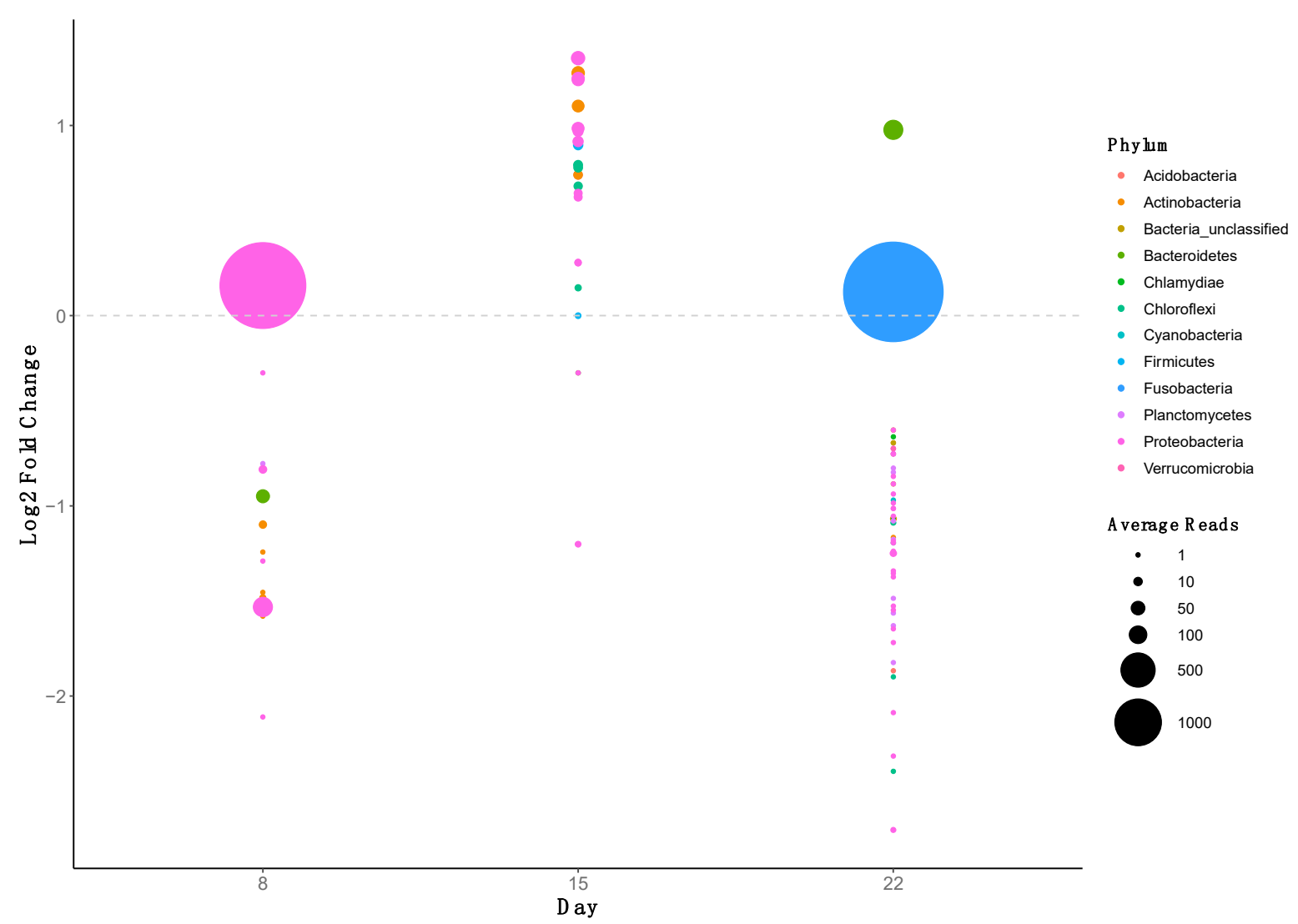

Figure 5. Plot of operational taxonomic units (OTU) that were significantly differentially abundant $(p<0.05)$ in the distal gut of Nile tilapia after treatment with oxytetracycline, compared with the control fish. Effect size is represented as the log2 fold-change of each OTU observed in fish from the oxytetracycline diet treatment compared with fish fed the control diet. Each circle represents a single OTU and is coloured according to the phylum to which the OTU originates. The circle size is proportional to the mean read abundance of each OTU.

\subsection{ARG Dynamics}

tet $A$ was found to dominate the distal gut of fish on day 0 , followed by intI1, where they represented $96 \%$ and $3 \%$ of the total ARG sequences detected, respectively (Figure $6 \mathrm{~A}$ ). At the end of antibiotic treatment, tet $A$ became enriched in the distal guts of the fish and in the tanks assigned to the OTC group, compared with the control group. In fact, on day 8 , tet $A$ made up $99 \%$ and $57 \%$, versus $34 \%$ and $8 \%$ of the ARGs detected in OTC and controltreated fish and tank biofilms, respectively (Figure 6A,B). In contrast, intI1 dominated the fish and tank biofilm assigned to the control group on day 8, where it represented $60 \%$ and $83 \%$, respectively. In addition, tet $M$ was also found more frequently in the control fish on day 8 compared with the OTC-treated fish, representing $6 \%$ and $<0.01 \%$ of the total ARGs detected, respectively. By day 15, the prevalence of intI1 and tetX increased in the OTC-treated fish, making up $27 \%$ and $48 \%$ of the total ARGs detected, respectively. Similar trends in tet $X$ were also found in the control fish on day 15 , where this gene represented $18 \%$ of the total ARGs detected. By day 22, tet $A$ dominated ( $>99 \%$ ) the ARGs detected within the distal guts of the OTC-treated fish. In comparison, intI1 and tet $A$ represented $29 \%$ and $71 \%$ of the ARGs detected in the distal guts of the control fish, respectively. All of the ARGs were also detected within the aquarium biofilter on day 0 (Figure 6C). Compared with the tank biofilms, the aquarium biofilter unit was more resilient to OTC treatment, as little variation was found in the distribution of ARGs over time. Likewise, all of the ARGs were also detected within most of the diet samples (Figure 6D). In addition, the distribution of ARGs detected was also fairly uniform across three out of five diet samples. 
More specifically, tet $A$ was undetectable within the control diet on day 8 , whereas tetX dominated the ARG profile of the diet given on day 22 .

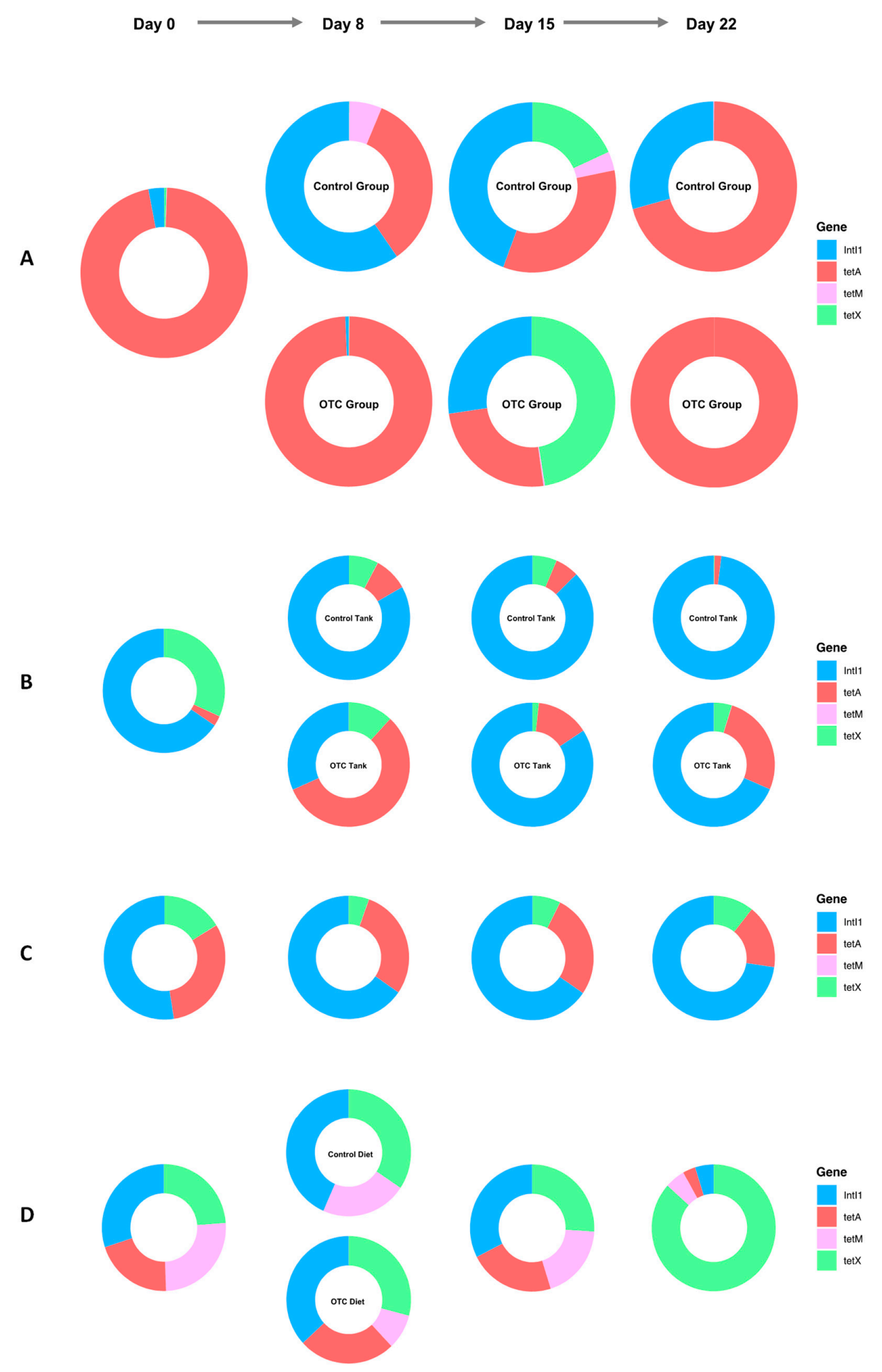

Figure 6. Distribution of intI1, tet $A$, tet $M$, and tet $X$ antimicrobial resistance genes in the distal guts of fish (A), tank biofilms (B), aquarium biofilter (C), and diets (D) before and after antibiotic treatment. 


\subsection{Correlation Analysis of ARGs and the Gut Microbiome in Fish}

ARGs intI1 and tet $M$ were both positively correlated with microbiome community diversity and evenness $(p<0.01)$ (Table 3$)$. While microbiome community evenness was positively associated with tet $X$ abundance $(p<0.05)$, microbiome community diversity was only found to have weak correlations with this gene $(p>0.05)$. The abundance of tet $A$ was not observed to have a strong correlation with any alpha diversity measure $(p>0.05)$.

Table 3. Associations between antimicrobial resistance gene (ARG) abundance and alpha diversity measures in the distal gut microbiome of Nile tilapia.

\begin{tabular}{ccccc}
\hline \multirow{2}{*}{ ARG } & \multicolumn{2}{c}{ Inverse Simpson } & \multicolumn{2}{c}{ Shannon Diversity Index } \\
\cline { 2 - 5 } & $\mathbf{R}$ & $\boldsymbol{p}$ & $\mathbf{R}$ & $\boldsymbol{p}$ \\
\hline intI1 & 0.58 & 0.0008 & 0.57 & 0.0011 \\
tet $A$ & 0.2 & 0.3000 & 0.24 & 0.2000 \\
tet $M$ & 0.69 & 0.0005 & 0.55 & 0.0092 \\
tet $X$ & 0.21 & 0.3700 & 0.45 & 0.0470 \\
\hline
\end{tabular}

R-Pearson's correlation coefficient.

A total of 109 OTUs were identified to be associated with ARG levels in the distal gut microbiome of Nile tilapia, where the magnitude of correlation ranged from -0.56 to -0.63 and 0.50 to 0.95 (Figure 7). The most diverse associations were found with intI1, where 55 OTUs $(50.46 \%)$ had a strong positive correlation with the abundance of this gene. tet $A$ was found to have the least diverse association with the microbiome community, as only five OTUs (4.58\%) were found to be strongly correlated with this ARG. At the phylum level, Actinobacteria, Planctomycetes, and Proteobacteria were found to have the greatest associations with the ARGs detected, as strong correlations between these genes and the OTUs assigned to these phyla were repeatedly detected. Within the Actinobacteria phylum, 15, 14, and 12 OTUs were found to be positively associated with the abundance of intI1, tet $M$, and tetX, respectively. These OTUs were assigned to several genera, including Mycobacterium, Nocardia, and Smaragdicoccus. Within Smaragdicoccus, OTU0063 was found to be positively associated with all three ARGs. A total of eight, six, and five Planctomycetes OTUs were shown to have strong positive correlations with intI1, tet $M$, and tet $X$ abundance, respectively. Within this phylum, most OTUs were assigned to uncultured taxa; however, OTU0262 and OTU0073 assigned to Gemmata and Planctopirus, respectively, were found to be positively associated with intI1. Additionally, two uncultured Planctomycete OTUs assigned to the Gemmataceae (OTU0165) and Pirellulaceae (OTU0221) families were also found to be associated with intI1, tet $M$, and tetX. A total of 23, 21, and 16 Proteobacteria OTUs were found to have positive correlations with the abundance of intI1, tet $M$, and tet $X$, respectively. These positively correlated OTUs were assigned to a range of genera, including Edwardsiella and Legionella, among others. Likewise, the OTUs assigned to Aquicella (OTU0044), Pedomicrobium (OTU0503), and Reyranella (OTU0006) were also identified as having positive associations with tet $M$, tet $X$, and intI1, respectively. Among the five OTUs strongly correlated with tet $A$ abundance, four were classified as Proteobacteria, including OTU0004, which contributed the majority of reads assigned to Plesiomonas. Several other OTUs belonging to other taxonomic groups were also identified as having positive associations with ARGs. These included those assigned to DS-100_ge (intI1), Dadabacteriales_ge (intI1), Flavobacterium (tetM and tetX), Mycoplasma (intI1), and Saccharimonadales_ge (intI1, tetM, and tetX). Negative correlations were observed between the abundance of OTU0001 assigned to Cetobacterium, which dominated the gut microbiome communities of fish, and the ARGs intI1 and tetM. 


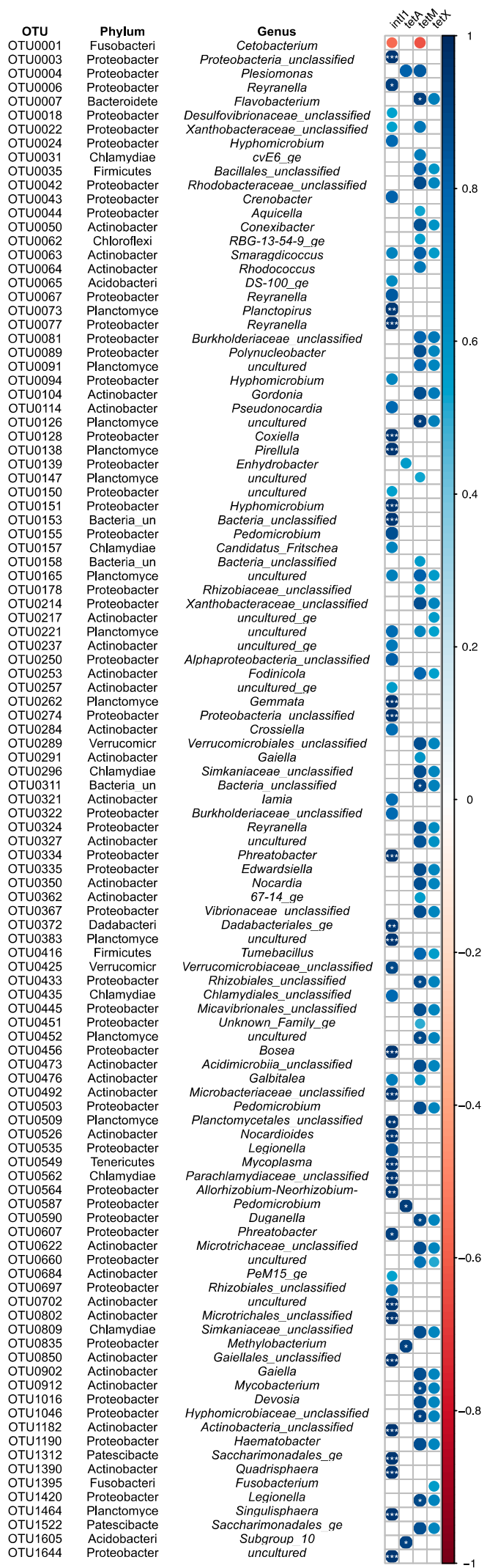

Figure 7. Correlation analysis between antimicrobial resistance gene abundance and operational taxonomic unit (OTU). Circle colour indicates negative or positive Pearson's correlation coefficient according to colour scale. ${ }^{*}$ denotes significance level e.g., ${ }^{*} p<0.05 ;{ }^{* *} p<0.001 ;{ }^{* * *} p<0.0001$. 


\section{Discussion}

Antibiotic treatment is a common husbandry practice used to treat and control the severity of infectious bacterial disease outbreaks in aquaculture. Moreover, this approach is of critical importance for farmed fish species like Nile tilapia, where alternative prophylactic strategies such as commercial vaccines are limited [35]. A recent culture-independent analysis revealed that antibiotic treatment can disrupt the gut microbiome, while promoting AMR within terrestrial vertebrate animals [36,37]. As members of the gut microbiome community serve vital biological functions for the fish host, understanding the intentional and unintentional consequences of antibiotic treatment on the gut microbiome community in farmed fish species is vital to support the overall health and welfare of the farmed animals.

In the study presented here, OTC at $100 \mathrm{mg} / \mathrm{kg} /$ day was not found to significantly affect overall growth performance of fish, although treated fish did have lower body weights and growth rates compared with the control group by the end of the trial. This was in agreement with findings from previous studies in Nile tilapia where fish fed OTC at $80 \mathrm{mg} / \mathrm{kg} /$ day, were not found to differ significantly in their growth performance compared with the control fish [17,38]. However, as OTC at 80 and $100 \mathrm{mg} / \mathrm{kg} /$ day has also been shown to significantly reduce and increase biomass in Nile tilapia when administered for 35 days and 12 weeks, respectively [39,40], it is likely that the lower biomass and growth rate of the treated fish compared with the control fish in the present study could become significant if the length of the treatment period was increased in future studies. Reduced growth performances in Nile tilapia treated with OTC has been previously attributed to reduced feed efficiency and nutrient digestibility through reductions in the protein and lipase enzyme activity, among other pathways [17,39]. Likewise, antibiotic-induced changes in the gut microbiome has also been implicated as an underlying mechanism in biomass changes within terrestrial vertebrate animals [41], and thus similar interactions may occur in fish.

Oxytetracycline treatment did not significantly affect bacterial diversity and evenness or community structure within the distal guts of the fish. These findings would therefore demonstrate a certain degree of resistance in the gut microbiome community of Nile tilapia in response to a single OTC exposure, and agree with a similar study in this fish species [17]. The microbiomes of both baseline and post-OTC treated fish in this study were dominated by Fusobacteria, supporting previous findings that members of this phylum are conserved in fish species, where they play a role in vitamin production for the fish host [19,25,42-44]. Resilience and enrichment in Fusobacteria following antibiotic treatment has been reported in zebrafish (Danio rerio) when exposed to OTC at similar or lower levels, respectively $[45,46]$. Therefore, the apparent resilience in the gut microbiome of fish in this study towards OTC could be explained by the predominance of this bacterial phyla.

Whilst the overall gut microbiome diversity and structure was resilient to OTC treatment, the abundance of numerous bacteria were found to significantly change following OTC exposure. Indeed, the most significant decrease in abundance was detected in the guts of the treated fish following OTC treatment at day 8 , similar to what has been reported for this antibiotic in Atlantic salmon [13,47]. This was particularly evident for the Gramnegative Proteobacteria OTUs assigned to Aeromonas and Reyranella. Furthermore, OTUs belonging to these genera remained depleted in OTC-treated fish even after a two-week withdrawal period. A general decline in members of the Proteobacteria phylum was not surprising given that OTC is frequently used in the treatment of bacterial fish pathogens, many of which belong to Proteobacteria [48-50] (p. 9). However, OTC was also shown to decrease the abundance of several Gram-positive bacteria, including those assigned to Lamia and other Actinobacteria genera. These findings therefore demonstrate the diverse nature of organisms within the gut microbiome, which can be unintentionally targeted by OTC during antibiotic treatment on the fish farm.

The statistically significant increase in Plesiomonas OTU at the end of OTC treatment suggests that this genus is resistant to some antibiotic compounds, and that OTC may promote the growth of Plesiomonas in the fish gut. This is consistent with results from a 
study in pacu following treatment with florfenicol [34]. Plesiomonas is a Gram-negative member of the Enterobacteriaceae family, which has been previously detected within the gut microbiome of numerous fish species including discus fish (Symphysodon haraldi) and zebrafish [51,52]. Recently, Plesiomonas shigelloides, the only species within this genus, was associated with clinical disease outbreaks in fish [53], therefore results from the present study indicate that OTC treatment may promote favourable growth conditions for resistant opportunistic pathogens already established within the fish gut microbiome. This agrees with the findings from a previous study in which Aeromonas salmonicida, the aetiological agent of furunculosis, was found to dominate the gut of Atlantic salmon following OTC treatment [13]. In this study, OTUs were only classified to genus level, however the association found between Plesiomonas and OTC treatment warrants further investigation to evaluate the impact of OTC treatment and the onset of disease.

One hypothesis for the resistance of Plesiomonas in this study is that it could be acquired, as tet $A$, an ARG that encodes for a tetracycline efflux pump, became enriched in the distal guts of OTC-treated fish following OTC treatment. This is in agreement with previous studies that detected tet $A$ in Plesiomonas from aquatic environments and fish including Nile tilapia [54-56]. Furthermore, the correlation analysis identified positive correlations between the abundances of the Plesiomonas OTU and the ARGs tetA and tetM, the latter of which encodes for a ribosomal protection protein. The correlation analysis also identified other positive associations between ARGs and resident microbiome members. For example, several correlations were found between the abundance of Actinobacteria members and ARGs, including a significant positive association between Mycobacterium and tet $M$ abundances. These findings were not surprising given that the first tetracycline compounds originated from Streptomyces aureofaciens, another member of the Actinobacteria phylum [57]. As such, in addition to producing antimicrobial compounds, Actinobacteria members may also contain a range of mechanisms that aid in defending against their own antibiotics, as well as resistance to compounds excreted from similar organisms. In fact, tet $M$ has previously been detected in several Actinobacteria genera, including Mycobacterium [58]. Taken together, the results from this study therefore suggest that OTC treatment may select for AMR within the gut microbiome of Nile tilapia, and supports findings from a previous study in Atlantic salmon [13]. These findings are a concern for the aquaculture industry, as the promotion of AMR within the gut microbiome from previous antibiotic exposures may reduce the effectiveness of future treatments with the same compound. This would be problematic for a number of fish farming countries, where only a small number of antibiotic compounds are licensed for use in farmed fish [50] and, as such, can be given multiple times during the production cycle.

As the fish used in this study had never previously received any antibiotic treatment, the detection of all four ARGs within the distal gut of the baseline fish was unexpected. The results could likely be explained by the colonisation of resistant microbial communities from the surrounding tank biofilm, as environmental microbiome communities are thought to colonise the developing fish gut during microbiome establishment [59]. This hypothesis is supported by the fact that the distal guts of fish and tank biofilms in this study shared several OTUs in common (data not shown). Moreover, all four ARGs investigated in this study were also detected in the tank biofilms and main biofilter unit within the aquarium. As the egg-associated microbiome community also facilitates the initial colonisation of the gut microbiome in fish [60], vertical transmission processes may have also played a role in the presence of ARGs in the baseline fish, similar to what has been reported for higher vertebrate animals [61]. In this study, the mouthbrooding behaviour of Nile tilapia may have allowed OTC-resistant bacteria carrying tetracycline ARGs within the maternal oral microbiome to transfer onto the egg surface, where they were able to colonise the distal guts of developing fish. However, to date, little is known about the vertical transmission of microbiome communities and their ARGs in fish.

This study had some limitations that should be considered when interpreting the findings. First, as non-invasive methods are currently not available or well evaluated for 
fish gut microbiome studies, we were unable to analyse the microbiome communities of individual fish over time. Whilst the sampling approach used in the present study is in line with other fish gut microbiome research [62,63], the development of non-terminal gut microbiome sampling methods in fish is warranted in order to improve the design of longitudinal studies. Secondly, although inter-individual variability in microbiome communities is well documented in fish and other vertebrate animals [64-68], the high individual variability between fish in this study may have masked any significant effects of OTC on growth performance and microbiome diversity. Whilst this study used a similar sample size to that of other fish microbiome studies $[17,27,39,69]$, future studies would benefit from increased sample sizes so as to further explore the findings from this study. However, this number must not compromise the ethical standards of the experiment and should be in agreement with the national regulations for animal research. Finally, the analysis of microbiome communities was performed via 16S rRNA amplicon sequencing, which is reliant on deposited sequences in curated databases. Furthermore, the short read length and high sequence similarity between certain taxonomic groups also gives this method poor discriminatory power below genus level [70]. As such, to build on the findings from this study, shotgun metagenomic methods that profile whole microbial genomes are warranted to identify the taxonomic and functional changes in the microbiome at a species/strain level, as well as to detect changes in a more diverse array of ARGs following antibiotic treatment.

\section{Materials and Methods}

\subsection{Experimental Design}

The effects of OTC exposure on the distal gut microbiome and ARG abundance in Nile tilapia was performed over a 36-day time series feeding study, which took place within the aquarium facilities at the Institute of Aquaculture (IoA), University of Stirling, UK. A total of 42 mixed sex, apparently healthy Nile tilapia (mean individual weight and lengths were $48.33 \pm 7.26 \mathrm{~g}$ and $13.69 \pm 0.76 \mathrm{~cm}$, respectively) were obtained from a single full-sib stock population held onsite at IoA. None of the fish had received any antibiotic treatment or vaccination prior to the start of the trial. The fish were randomly allocated into individual $19 \mathrm{~L}$ tanks, which were maintained on a recirculation system, at a flow rate of $1.2 \mathrm{~L} / \mathrm{min}$, under a 12:12 h light:dark cycle, and ambient water temperature of $27 \pm 0.5^{\circ} \mathrm{C}$. Fish were maintained in these conditions throughout the entire trial.

Following a 14-day acclimation period, the tanks were randomly allocated into two treatment groups $\left(\mathrm{n}_{\text {tanks }}=18\right.$ per treatment). Fish in treatment group one were fed a medicated diet, surface coated with OTC at $100 \mathrm{mg} / \mathrm{kg} /$ day to reflect a dose that has been reported in aquaculture [17]. Furthermore, when given at a similar or lower dose, OTC has been shown to improve growth and feed efficiency, as well as alter the gut microbiome in Nile tilapia, respectively [17,40]. Fish in treatment group two were fed a non-medicated (control) diet. Both diets were delivered into respective tanks at a rate of $1.5 \%$ bodyweight/day for eight days. After the 8-day treatment period, fish in both treatment groups were fed the control diet at a feeding rate of $1.5 \%$ bodyweight/day for 14 days, after which time the experimental trial was terminated. The feeding rate was chosen based on advice from veterinary staff and aquarium technicians following monitoring of the feeding response of fish during the acclimation period. Throughout the entire trial, diets were split into morning and afternoon rations for each fish, which were fed by hand. During each feeding period, the feeding response of individual fish was monitored to ensure the complete diet allocation was consumed.

\subsection{Diet Preparation and In Vitro Antimicrobial Testing}

The commercial pelleted feed Standard Expanded Floating Pellet $3 \mathrm{~mm}(8 \%$ oil content, $40 \%$ protein content) (Skretting, Wincham, UK) was used throughout the experiment. The OTC diet was prepared by surface coating pellets with OTC hydrochloride ( $98.2 \%$ purity) (Duchefa Biochemie ${ }^{\circledR}$, Haarlem, The Netherlands). Following the coating of pellets with 
OTC, cod liver oil (Vitarenew ${ }^{\circledR}$; Principle Healthcare International Limited, Skipton, UK) was applied at a rate of $20 \mathrm{~mL} / \mathrm{kg}$ diet to bind the antibiotic to the pellets. The OTC diet was prepared $24 \mathrm{~h}$ prior to commencing the treatment period. All diets were stored at $4{ }^{\circ} \mathrm{C}$ until required.

Prior to feeding the fish, the OTC diet was tested for antimicrobial activity against the OTC-sensitive $A$. hydrophila NCIMB 9240. Briefly, a bacterial suspension was made using a colony of $A$. hydrophila inoculated into $30 \mathrm{~mL}$ sterile tryptone soy broth (Oxoid ${ }^{\circledR}$, Basingstoke, UK) and was incubated for $18 \mathrm{~h}$ at $28^{\circ} \mathrm{C}$. Following incubation, the bacterial suspension was centrifuged at $2600 \times g$ for $15 \mathrm{~min}$ at $4{ }^{\circ} \mathrm{C}$. The resulting bacterial pellet was then resuspended in sterile phosphate buffered saline $(\mathrm{pH}$ 7.2) to reach a MacFarland standard equivalent of 5.0, as judged by the naked eye. Then, a bacterial lawn containing a total of $100 \mu \mathrm{L}$ of the bacterial suspension was spread onto sterile tryptone soy agar (Oxoid ${ }^{\circledR}$, Basingstoke, UK). This was left for $5 \mathrm{~min}$ at room temperature, after which, three pellets from the OTC diet were then aseptically placed onto the agar plate. All pellets were placed carefully to ensure they remained separated. The agar plate was then sealed before incubating at $28^{\circ} \mathrm{C}$ for $48 \mathrm{~h}$. Bacterial growth and zones of inhibition around the diet pellets were recorded after $48 \mathrm{~h}$. Pellets from the control diet were also tested to confirm they were free from any antimicrobial compounds.

\subsection{Sample Collection}

Gut digesta was aseptically collected from individual fish at four time points, which were as follows: immediately before antibiotic treatment (day 0; baseline), at the end of the antibiotic treatment (day 8), one-week post-treatment withdrawal (day 15), and at the end of the two-week withdrawal period (day 22). At each timepoint, individual fish from six tanks were randomly sampled from each treatment group, giving $n=6$ fish per treatment group and per sampling time point. This followed the international recommendations for RNA-seq experiments [71], which use similar molecular methods, and met conditions for the 3Rs framework in animal research [72]. Following euthanasia by a lethal dose of tricaine methanesulfonate (1000 mg/g; Pharmaq ${ }^{\circledR}$, Fordingbridge, UK), the fish were weighed and the total length (snout to caudal fin) recorded. Following this, the digesta from individual fish was aseptically collected from the distal portion of the gut (distal point of midgut to $\sim 2 \mathrm{~cm}$ before the vent), as described by [73], except gut digesta was stored in empty $2 \mathrm{~mL}$ microcentrifuge tubes (Alpha Labs ${ }^{\circledR}$, Eastleigh, UK). No intact feed pellets were observed within the digesta of any of the fish sampled. In addition to the gut digesta samples, a total of ten pellets from each diet (stored in sterile $7 \mathrm{~mL}$ containers) and biofilm samples from the main filtration unit, as well as a random tank for each treatment/time point, were also collected. Biofilm samples were collected using a sterile swab (VWR International, Monroeville, PA, USA) placed just below the water line and moved around each side of the tank/filtration unit for ca. $20 \mathrm{~s}$. Biofilm samples were stored in $2 \mathrm{~mL}$ microcentrifuge tubes as described for the digesta samples. At each sampling point, all samples were held on ice until sampling was complete, and they were stored at $-80^{\circ} \mathrm{C}$ until required.

\subsection{Library Preparation and Illumina Miseq Sequencing}

A total of $162.9 \pm 77.7 \mathrm{mg}$ of gut digesta was processed for genomic DNA extraction, following the protocol described by [74], using the QIAamp Fast DNA Stool Mini Kit (Qiagen ${ }^{\circledR}$, Manchester, UK) and 0.7 mm garnet beads (PowerBead Tubes, Qiagen ${ }^{\circledR}$, Manchester, UK). The final DNA was eluted in a $35 \mu \mathrm{L}$ EB buffer (10 mM Tris-HCl, pH 8.5; Qiagen ${ }^{\circledR}$, Manchester, UK). The concentration and purity of the eluted DNA samples was measured using the Nanodrop ${ }^{\circledR}$ 2000c spectrophotometer (ThermoFisher Scientific, Basingstoke, UK) and ten microlitre aliquots, and was stored at $-20{ }^{\circ} \mathrm{C}$ until required. Genomic DNA was also extracted from the diet pellets $(80 \mathrm{mg}$; ca. six pellets) and biofilm samples using the same commercial DNA extraction kit and method described previously. Genomic DNA was extracted for a group of NSCs in an attempt to track all sources of microbial DNA contamination in $16 \mathrm{~S}$ rRNA libraries. No sample or DNA was added to 
the NSC samples, instead, the inhibitEX buffer supplied in the DNA extraction kit was used as the starting material. The NSC samples were generated for all starting material types, including the digesta (NSC_Fish), diet (NSC_Diet), and biofilm (NSC_Tank) samples, respectively. The NSC_Tank sample included a sterile swab similar to that used in the original sampling. Lastly, a mock microbiome community (IoA_MB_STD) was generated for use as an internal sequencing control. The IoA_MB_STD sample contained genomic DNA at equal concentrations from five bacterial species known to colonise fish, including A. hydrophila NCIMB 9240, Edwardsiella ictaluri NCIMB 13272, Pseudomonas aeruginosa ATCC 27853, Vibrio anguillarum NCIMB 6, and Yersinia ruckeri NCIMB 2194.

Prior to preparing 16S rRNA Illumina libraries, the bacterial DNA yield recovered from the gut digesta and NSC samples was quantified using TaqMan real-time qPCR methods and the primer/probe combination listed in Table 4. Real-time qPCR with absolute quantification was performed on a Stratagene Mx3005P QPCR System (Agilent Technologies LDS UK Ltd., Cheshire, UK). Quantitative analysis of the 16S rRNA gene copy number was performed in triplicate $20 \mu \mathrm{L}$ reactions containing the following: $10 \mu \mathrm{L}$ SensiFAST ${ }^{\mathrm{TM}}$ Probe Lo-ROX mastermix (Bioline Reagents Limited, London, UK), $0.4 \mu \mathrm{L}$ of each forward and reverse primer $(0.2 \mu \mathrm{M})$ (Eurofins Biomnis UK Ltd., Guildford, UK), $0.1 \mu \mathrm{L}$ probe $(0.05 \mu \mathrm{M})$ (Eurofins Biomnis UK Ltd., Guildford, UK), 7.1 $\mu \mathrm{L}$ nuclease-free water, and $2 \mu \mathrm{L}$ DNA $(<50 \mathrm{ng} / \mu \mathrm{L})$. Duplicate no DNA template control (NTC) reactions were also included in every qPCR run to confirm qPCR reagents were free from microbial DNA contamination. Real-time qPCR conditions were as follows: an initial denaturation step at $95^{\circ} \mathrm{C}$ for ten minutes, followed by $40 \times$ cycles at $95^{\circ} \mathrm{C}$ for $30 \mathrm{~s}$ and $60{ }^{\circ} \mathrm{C}$ for one minute. The number of $16 \mathrm{~S}$ rRNA genes per microlitre of DNA sample was calculated from the final $\mathrm{Ct}$ values in each qPCR reaction using a standard curve. The standard curve was generated using plasmid DNA containing the 16S rRNA V3-4 hypervariable region insert. Briefly, plasmid DNA standards were generated using the pGEM-T Easy Vector system (Promega Corporation, Fitchburg, WI, USA) and were transformed into Escherichia coli XL1-Blue cells (recA1 endA1 gyrA96 thi-1 hsdR17 supE44 relA1 lac (F' proAB lacIqZAM15 Tn10 [Tetr])) (Agilent Technologies Inc, Cheshire, UK), following the manufacturer's protocol. The plasmid standards were ten-fold serially diluted to concentrations from $1 \times 10^{8}$ to $1 \times 10^{3} 16 \mathrm{~S}$ rRNA gene copies/ $\mu \mathrm{L}$. The $\mathrm{qPCR}$ efficiencies and $\mathrm{R}^{2}$ values are detailed in Table 4.

Table 4. Primer sets used in this study.

\begin{tabular}{|c|c|c|c|c|c|c|c|c|}
\hline Primer & Target & Sequence $\left(5^{\prime}-{ }^{\prime} 3\right)$ & Size (bp) & $\mathrm{Ta}^{\circ} \mathrm{C}$ & Eff. (\%) & $\mathbf{R}^{2}$ & Application & Source \\
\hline $\begin{array}{l}341 \mathrm{~F} \\
805 \mathrm{R} \\
\text { Probe }\end{array}$ & 16S rRNA (V3-4) & $\begin{array}{c}\text { CCTACGGGNGGCWGCAG } \\
\text { GACTACHVGGGTATCTAATCC } \\
\text { FAM-ATTACCGCGGCTGCTGG-MGBEO }\end{array}$ & 464 & 60 & 114.37 & 0.99 & $\begin{array}{l}\text { 16S rRNA } \\
\text { qPCR }\end{array}$ & {$[75,76]$} \\
\hline$\overline{1} \overline{\mathrm{S}} \_\overline{\mathrm{V}} \overline{\mathrm{F}} \mathrm{F}$ & & [Illumina adapter]-AYTGGGYDTAAAGN & . & & & & & \\
\hline $\begin{array}{l}\text { 16S_V4R } \\
\text { Cocktail }\end{array}$ & 16S rRNA (V4) & $\begin{array}{c}\text { [Illumina adapter] } \\
\text { TACCRGGGTHTCTAATCC } \\
\text { [Illumina } \\
\text { adapter]-TACCAGAGTATCTAATTC } \\
\text { [Illumina } \\
\text { adapter]-CTACDSRGGTMTCTAATC } \\
\text { [Illumina } \\
\text { adapter]-TACNVGGGTATCTAATC }\end{array}$ & 245 & 54 & $\mathrm{~N} / \mathrm{A}$ & $\mathrm{N} / \mathrm{A}$ & $\begin{array}{l}\text { Illumina } \\
\text { Libraries }\end{array}$ & [77] \\
\hline $\begin{array}{l}\text { intII_- } \bar{F} \\
\text { intI1 R }\end{array}$ & Class 1 integrase protein & CСТCCСGCACGATGATC & 280 & 63 & 101 & 0.99 & ARG qPCR & {$[78]$} \\
\hline $\begin{array}{l}\text { tetA_F } \\
\text { tetA_R }\end{array}$ & Tetracycline efflux pump & CATAGATCGCCGTGAAGAGG & 210 & 64 & ---- & $\begin{array}{c}--- \\
0.99\end{array}$ & ARG qPCR & --- \\
\hline $\begin{array}{l}\text { tet } \bar{M} \_\bar{F} \\
\text { tetM_R }\end{array}$ & $\begin{array}{l}\text { Tetracycline ribosomal } \\
\text { protection }\end{array}$ & 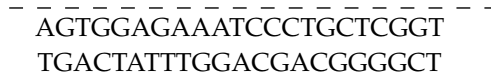 & 149 & 66 & 107 & $\begin{array}{ll}-- \\
0.99\end{array}$ & ARG qPCR & {$[-\overline{-}-\bar{c}$} \\
\hline $\begin{array}{l}\text { tetX_- } \bar{F} \\
\text { tetX_R }\end{array}$ & $\begin{array}{l}\text { Enzymatic modification of } \\
\text { tetracycline }\end{array}$ & $\begin{array}{l}\text { GAAA AAGACAACGACCGAGAG }- \\
\text { ACACCATTGGTAAGGTAAG }\end{array}$ & 131 & 63 & --- & 0.99 & ARG qPCR & $\overline{[78}]$ \\
\hline
\end{tabular}

Ta; Annealing temperature. ARG; antimicrobial resistance gene. 
The bacterial 16S rRNA V4 hypervariable region was amplified using the 16S_V4F and 16S_V4R cocktail primers listed in Table 4. All of the samples were amplified in triplicate $10 \mu \mathrm{L}$ reactions using $5 \mu \mathrm{L} 2$ X NEBNext Ultra II Q5 mastermix (New England Biolabs (UK) Ltd., Hitchin, UK), with $0.4 \mu \mathrm{L}$ of each primer $(0.2 \mu \mathrm{M})$ (Eurofins Biomnis UK Ltd., Guildford, UK), $0.2 \mu \mathrm{L}$ nuclease-free water and $4 \mu \mathrm{L}$ DNA $\left(1.49 \times 10^{4} 16 \mathrm{~S} \mathrm{rRNA}\right.$ copies $\left./ \mu \mathrm{L}\right)$. Amplification was conducted in a Tgradient thermal cycler (Biometra GmbH, Göttingen, Germany) under the following conditions: $98^{\circ} \mathrm{C}$ for $2 \mathrm{~min}$, followed by $30 \times$ cycles of $98^{\circ} \mathrm{C}$ for $15 \mathrm{~s}, 54{ }^{\circ} \mathrm{C}$ for $30 \mathrm{~s}$, and $65^{\circ} \mathrm{C}$ for $45 \mathrm{~s}$. All PCR reactions underwent a final extension stage at $65{ }^{\circ} \mathrm{C}$ for $10 \mathrm{~min}$. The generated libraries were purified using the AxyPrep Mag PCR clean up Kit (Appleton Woods Ltd., Birmingham, UK), following the manufacturer's protocol, except with a modified 1:1 volume of library to magnetic beads. A total of $7 \mu \mathrm{L}$ of each library was indexed using the Nextera XT index primers N7XX and S5XX (Illumina ${ }^{\circledR}$, San Diego, CA, USA). Libraries were also generated for the IoA_MB_STD sample, as well as for all NSC samples. The final libraries (length $\sim 381 \mathrm{bp}$ ) were sequenced using the Illumina MiSeq ${ }^{\circledR}$ NGS system with the Illumina ${ }^{\circledR}$ MiSeq Reagent Kits v2 $(2 \times 250$ bp; 500-cycle) (Illumina ${ }^{\circledR}$, San Diego, CA, USA) at IoA.

\subsection{Bioinformatic Analysis of Illumina Miseq Data}

Raw Illumina reads were demultiplexed with Casava v. 1.8 (Illumina ${ }^{\circledR}$, San Diego, CA, USA), and reads representing the PhiX/internal controls or reads not matching the Illumina indices were removed. The open-source program Mothur [79] was used to process the sequence read data generated. Reads were first quality-filtered to remove sequences that contained ambiguous bases, homopolymers longer than $8 \mathrm{bp}$, and reads with sequences less than $235 \mathrm{bp}$ or more than $250 \mathrm{bp}$. The reads were then further denoised, allowing for up to $2 \mathrm{bp}$ differences between the duplicate sequences. The reads were assessed for chimeric sequences [80], which were then discarded before the remaining reads were aligned to the SILVA-based bacterial reference alignment [Release 132, December 2017] [81]. Any reads assigned to undesired lineages including "chloroplast", "mitochondria", "archaea", "eukaryota", or "unknown" were later discarded. The sequence reads associated with the IoA_MB_STD sample were used to calculate the sequence error rate and were then removed from the final dataset. Then, OTUs were selected using the cluster.split command and a sequence cut-off of $97 \%$, and were classified using the SILVA database described above. Finally, singleton OTUs were removed from the final dataset. The final dataset was then rarefied to 18,145 sequences, the lowest number of sequences per sample, prior to performing any further downstream analysis. The final sample size after rarefaction was $n=5$ for day 0 , baseline; $n=4, n=3$ for the control and OTC groups at day 8 , respectively; $n=4, n=5$ for the control and OTC groups at day 15 , respectively; and $n=6, n=4$ for the control and OTC groups at day 22, respectively.

\subsection{Quantification of $A R G$ s}

Real-time qPCR with absolute quantification was used to quantify the abundance of four ARGs, previously used to monitor AMR within DNA samples derived from aquatic environments [78]. Target genes included a class 1 intregrase protein (intI1), as well as three tetracycline resistance genes (tet $A$, tet $M$, and tet $X)$ (Table 4). Quantification was performed in triplicate reactions for each DNA sample on the LightCycler ${ }^{\circledR} 480$ II platform (Roche Diagnostics Ltd, Burgess Hill, UK). The qPCR reactions were prepared to a total volume of $10 \mu \mathrm{L}$ containing $5 \mu \mathrm{L}$ Luminaris Color HiGreen qPCR Master Mix (ThermoFisher Scientific, Basingstoke, UK), $3 \mu \mathrm{L}$ nuclease-free water, $0.5 \mu \mathrm{L}$ of each forward and reverse primers $(0.5 \mu \mathrm{M})$ (Eurofins Biomnis UK Ltd., Guildford, UK), and $1 \mu \mathrm{L}$ DNA. The primer sequences, annealing temperatures, and expected amplicon sizes for each gene are listed in Table 4. Duplicate NTC reactions were included in every qPCR run. Quantification was performed following an initial denaturation step at $95^{\circ} \mathrm{C}$ for $10 \mathrm{~min}$, then $40 \times$ cycles at $95{ }^{\circ} \mathrm{C}$ for $15 \mathrm{~s}, \mathrm{n}{ }^{\circ} \mathrm{C}$ for $30 \mathrm{~s}$, and $72{ }^{\circ} \mathrm{C}$ for $30 \mathrm{~s}$. Finally, a gradient of $0.11{ }^{\circ} \mathrm{C}$ per second and five reads per ${ }^{\circ} \mathrm{C}$ from $72{ }^{\circ} \mathrm{C}$ to $95{ }^{\circ} \mathrm{C}$ was performed for the melt-curve analysis to 
confirm the specificity of the amplified qPCR products. The number of gene copies per microlitre of DNA samples was calculated from the final $\mathrm{Ct}$ values of each reaction, using a standard curve of serially diluted plasmid containing the respective gene insert from $1 \times 10^{8}$ to $1 \times 10^{1}$ gene copies $/ \mu \mathrm{L}$. The plasmids were prepared in E. coli strain DH5 $\alpha$ (fhuA2 $\Delta$ (argF-lacZ)U169 phoA glnV44 $\Phi 80 \Delta$ (lacZ)M15 gyrA96 recA1 relA1 endA1 thi-1 hsdR1) (ThermoFisher Scientific, Basingstoke, UK) using the PGEM T-Easy vector system described previously and a pool of DNA from the OTC tank biofilms at days 8, 15, and 22 as the template material. The gene copies for each sample were normalised to the $16 \mathrm{~S}$ rRNA gene copy number of the same sample. Gene copies were used as an indicator for the relative levels of ARGs within microbiome communities before and after antibiotic treatment. The efficiencies and $\mathrm{R}^{2}$ values for each qPCR assay are detailed in Table 4 .

\subsection{Data Visualisation and Analysis}

Data visualisation and statistical analysis were conducted in JMP ${ }^{\circledR}$ version 14 and Rstudio Version 1.1.419, using ggplot2 [82], phyloseq [83], reshape2 [84], and vegan [85] packages, respectively. Differences in the final mean length, weight, and growth rate (g/day) of the fish across treatment groups and time were evaluated using two-way ANOVA. Changes in alpha diversity following antibiotic treatment were measured using the Inverse Simpson's and the Shannon's diversity indices, which account for species diversity as well as richness and evenness, respectively. Differences in alpha diversity measurements were also evaluated using two-way ANOVA analysis, with treatment and time as factors. However, prior to performing the analysis, all alpha diversity data were $\log _{10}$ transformed to normalise the data distribution. Distance matrices of beta diversity were generated using the thetaYC coefficient [86] and Bray-Curtis dissimilarity [87] calculators on Mothur. For all distance measures, PERMANOVA (vegan; adonis function) [88] was first used to test the differences in beta diversity according to sample type, designated as fish (distal gut), tank biofilm, aquarium biofilter, diet, or NSCs. Following this, PERMANOVA was used to further test the influence of treatment and time on the inter-sample distances of the distal gut microbiome communities. PERMANOVA was conducted using 10,000 permutations. Correlations between microbiome diversity and the abundance of ARGs were calculated using Pearson's correlation coefficient. Furthermore, the degree of interaction and correlation between individual OTUs and ARGs was further assessed with Pearson's correlation coefficient using the rcorr.adjust algorithm provided in the RcmdrMisc package [89]. Significance of correlations was corrected for multiple inferences using Holm's method.

\section{Conclusions}

The findings from this study demonstrated that whilst the overall gut microbiome community of Nile tilapia remained resilient to a single treatment with OTC, the abundance of particular microbiome community members shifted. A significantly positive shift in Plesiomonas abundance was observed in OTC-treated fish following antibiotic treatment. Moreover, a number of Gram-negative and Gram-positive organisms significantly declined in abundance after antibiotic treatment, demonstrating the huge diversity of bacteria that can be unintentionally affected with a single application. Antibiotic treatment with OTC was also associated with an increase in the abundance of certain ARGs. Furthermore, a number of strong correlations were observed between members of the microbiome community, including Plesiomonas, and the abundance of several ARGs. Taken together, these findings demonstrated that antibiotic treatment does disrupt gut microbiome membership in fish, and is a potential pressure in AMR development in the recovered microbiome community. Further work is required to clarify the long-term consequences of antibiotic-induced changes in the gut microbiome and AMR development in this fish species.

Supplementary Materials: The following are available online at https://www.mdpi.com/article/10 .3390/antibiotics10101213/s1. Table S1: Operational taxonomic units (OTU) identified as discriminatory according to oxytetracycline exposure by Metastats and LEfSe algorithms in Mothur. 
Author Contributions: Conceptualization, C.J.P. and M.C.; methodology, C.J.P.; software, C.J.P.; formal analysis, C.J.P., J.F.T., S.M. and M.C.; investigation, C.J.P.; resources, C.J.P., S.M. and M.C.; data curation, C.J.P.; writing—original draft preparation, C.J.P.; writing-review and editing, C.J.P., J.F.T., S.M. and M.C.; supervision, S.M. and M.C.; project administration, M.C.; funding acquisition, C.J.P., S.M. and M.C. All authors have read and agreed to the published version of the manuscript.

Funding: This research was funded by Fisheries Society of the British Isles through their $\mathrm{PhD}$ studentship program and a small research grant.

Institutional Review Board Statement: The study was conducted according to the guidelines of the UK Home Office Animals (Scientific Procedures) Act 1986 and was approved by the Animal Welfare and Ethical Review Body at the University of Stirling (AWERB (18 19) 151 New ASPA).

Data Availability Statement: Sequence files and metadata for samples used in this study are available from the European Nucleotide Archive (ENA accession number: ERP131454).

Acknowledgments: The authors would like to thank aquarium staff for their technical assistance during the feeding trial. Thanks are also given to J. Taggart and J. Ireland for their expertise and guidance during the molecular phase of this research, as well as C. Broughton, F. Legario, and A. Ahmed for their help during the sample processing.

Conflicts of Interest: The authors declare no conflict of interest. The funders had no role in the design of the study; in the collection, analyses, or interpretation of data; in the writing of the manuscript; or in the decision to publish the results.

\section{References}

1. FAO-The State of World Fisheries and Aquaculture 2020. In Sustainability in Action; FAO: Rome, Italy, $2020 ;$ p. 21. ISBN 9789251326923.

2. FAO-The State of World Fisheries and Aquaculture 2018. In Meeting the Sustainable Development Goals; FAO: Rome, Italy, 2018; p. 23. ISBN 9789251305621.

3. Kobayashi, M.; Msangi, S.; Batka, M.; Vannuccini, S.; Dey, M.M.; Anderson, J.L. Fish to 2030: The Role and Opportunity for Aquaculture. Aquac. Econ. Manag. 2015, 19, 282-300. [CrossRef]

4. Ibrahem, M.; Mostafa, M.; Arab, R.M.; Rezk, M. Prevalence of Aeromonas hydrophila Infection in Wild and Cultured Tilapia Nilotica (O. niloticus). In Proceedings of the 8th International Symposium on Tilapia Aquaculture, Cairo, Egypt, 12-14 October 2008; pp. 1257-1271.

5. Soto, E.; Griffin, M.; Arauz, M.; Riofrio, A.; Martinez, A.; Cabrejos, M.E. Edwardsiella ictalurias the Causative Agent of Mortality in Cultured Nile Tilapia. J. Aquat. Anim. Health 2012, 24, 81-90. [CrossRef] [PubMed]

6. Jantrakajorn, S.; Maisak, H.; Wongtavatchai, J. Comprehensive Investigation of Streptococcosis Outbreaks in Cultured Nile Tilapia, Oreochromis niloticus, and Red Tilapia, Oreochromis sp., of Thailand. J. World Aquac. Soc. 2014, 45, 392-402. [CrossRef]

7. Zheng, Y.; Wu, W.; Hu, G.; Qiu, L.; Meng, S.; Song, C.; Fan, L.; Zhao, Z.; Bing, X.; Chen, J. Gut microbiota analysis of juvenile genetically improved farmed tilapia (Oreochromis niloticus) by dietary supplementation of different resveratrol concentrations. Fish Shellfish. Immunol. 2018, 77, 200-207. [CrossRef] [PubMed]

8. Monteiro, S.H.; Francisco, J.; Campion, T.; Pimpinato, R.; Andrade, G.; Garcia, F.; Tornisielo, V. Multiresidue antimicrobial determination in Nile tilapia (Oreochromis Niloticus) cage farming by liquid chromatography tandem mass spectrometry. Aquaculture 2015, 447, 37-43. [CrossRef]

9. Eissa, F.; Ghanem, K.; Al-Sisi, M. Occurrence and human health risks of pesticides and antibiotics in Nile tilapia along the Rosetta Nile branch, Egypt. Toxicol. Rep. 2020, 7, 1640-1646. [CrossRef] [PubMed]

10. Ström, G.H.; Björklund, H.; Barnes, A.C.; Da, C.T.; Nhi, N.H.Y.; Lan, T.T.; Magnusson, U.; Haldén, A.N.; Boqvist, S. Antibiotic Use by Small-Scale Farmers for Freshwater Aquaculture in the Upper Mekong Delta, Vietnam. J. Aquat. Anim. Heal. 2019, 31, 290-298. [CrossRef]

11. Yang, T.; Yang, K.; Chen, Y.; Fan, K. Characterization of a Bi-directional Promoter OtrRp Involved in Oxytetracycline Biosynthesis. Curr. Microbiol. 2019, 76, 1264-1269. [CrossRef]

12. Chopra, I.; Robers, M. Tetracycline Antibiotics: Mode of Action, Applications, Molecular Biology, and Epidemiology of Bacterial Resistance. Microbiol. Mol. Biol. Rev. 2001, 65, 232-260. [CrossRef] [PubMed]

13. Navarrete, P.; Mardones, P.; Opazo, R.; Espejo, R.; Romero, J. Oxytetracycline Treatment Reduces Bacterial Diversity of Intestinal Microbiota of Atlantic Salmon. J. Aquat. Anim. Health 2008, 20, 177-183. [CrossRef]

14. Favero, L.M.; Facimoto, C.T.; Chideroli, R.T.; da Costa, A.R.; Umezu, D.F.; Honda, B.T.B.; de Oliveira, A.G.; Flaiban, K.K.M.D.C.; Di Santis, G.W.; Pereira, U.D.P. Administration of dehydrated oxytetracycline effectively reduces francisellosis mortality in Nile tilapia. Aquac. Res. 2021, 52, 4116-4126. [CrossRef] 
15. Roy, A.; Abraham, T.J.; Namdeo, M.S.; Singha, J.; Julinta, R.B.; Boda, S. Effects of Oral Oxytetracycline-Therapy on Wound Progression and Healing Following Aeromonas caviae Infection in Nile Tilapia (Oreochromis niloticus L.). Braz. Arch. Biol. Technol. 2019, 62. [CrossRef]

16. Oviedo-Bolaños, K.; Rodríguez-Rodríguez, J.A.; Sancho-Blanco, C.; Barquero-Chanto, J.E.; Peña-Navarro, N.; Escobedo-Bonilla, C.M.; Umaña-Castro, R. Molecular identification of Streptococcus sp. and antibiotic resistance genes present in Tilapia farms (Oreochromis niloticus) from the Northern Pacific region, Costa Rica. Aquac. Int. 2021, 29, 2337-2355. [CrossRef]

17. Limbu, S.; Zhou, L.; Sun, S.-X.; Zhang, M.-L.; Du, Z.-Y. Chronic exposure to low environmental concentrations and legal aquaculture doses of antibiotics cause systemic adverse effects in Nile tilapia and provoke differential human health risk. Environ. Int. 2018, 115, 205-219. [CrossRef] [PubMed]

18. National Office of Animal Health NOAH Compendium. Available online: http://www.noahcompendium.co.uk (accessed on 29 August 2019).

19. Bereded, N.; Abebe, G.; Fanta, S.; Curto, M.; Waidbacher, H.; Meimberg, H.; Domig, K. The Impact of Sampling Season and Catching Site (Wild and Aquaculture) on Gut Microbiota Composition and Diversity of Nile Tilapia (Oreochromis niloticus). Biology 2021, 10, 180. [CrossRef] [PubMed]

20. Bereded, N.K.; Curto, M.; Domig, K.J.; Abebe, G.B.; Fanta, S.W.; Waidbacher, H.; Meimberg, H. Metabarcoding Analyses of Gut Microbiota of Nile Tilapia (Oreochromis niloticus) from Lake Awassa and Lake Chamo, Ethiopia. Microorganisms 2020, 8, 1040. [CrossRef]

21. Tan, H.Y.; Chen, S.-W.; Hu, S.-Y. Improvements in the growth performance, immunity, disease resistance, and gut microbiota by the probiotic Rummeliibacillus stabekisii in Nile tilapia (Oreochromis niloticus). Fish Shellfish. Immunol. 2019, 92, 265-275. [CrossRef]

22. Hallali, E.; Kokou, F.; Chourasia, T.K.; Nitzan, T.; Con, P.; Harpaz, S.; Mizrahi, I.; Cnaani, A. Dietary salt levels affect digestibility, intestinal gene expression, and the microbiome, in Nile tilapia (Oreochromis niloticus). PLoS ONE 2018, 13, e0202351. [CrossRef] [PubMed]

23. Zhang, M.; Sun, Y.; Liu, Y.; Qiao, F.; Chen, L.; Liu, W.-T.; Du, Z.; Li, E. Response of gut microbiota to salinity change in two euryhaline aquatic animals with reverse salinity preference. Aquaculture 2016, 454, 72-80. [CrossRef]

24. Parata, L.; Mazumder, D.; Sammut, J.; Egan, S. Diet type influences the gut microbiome and nutrient assimilation of Genetically Improved Farmed Tilapia (Oreochromis niloticus). PLoS ONE 2020, 15, e0237775. [CrossRef]

25. Sakyi, M.E.; Cai, J.; Ampofo-Yeboah, A.; Anokyewaa, M.A.; Wang, Z.; Jian, J. Starvation and re-feeding influence the growth, immune response, and intestinal microbiota of Nile tilapia (Oreochromis niloticus; Linnaeus 1758). Aquaculture 2021, 543, 736959. [CrossRef]

26. Carlson, J.M.; Leonard, A.B.; Hyde, E.R.; Petrosino, J.F.; Primm, T.P. Microbiome disruption and recovery in the fish Gambusia affinis following exposure to broad-spectrum antibiotic. Infect. Drug Resist. 2017, 10, 143-154. [CrossRef] [PubMed]

27. Zhou, L.; Limbu, S.M.; Shen, M.; Zhai, W.; Qiao, F.; He, A.; Du, Z.-Y.; Zhang, M. Environmental concentrations of antibiotics impair zebrafish gut health. Environ. Pollut. 2018, 235, 245-254. [CrossRef] [PubMed]

28. Zhao, S.; Ma, L.; Wang, Y.; Fu, G.; Zhou, J.; Li, X.; Fang, W. Antimicrobial resistance and pulsed-field gel electrophoresis typing of Vibrio parahaemolyticus isolated from shrimp mariculture environment along the east coast of China. Mar. Pollut. Bull. 2018, 136, 164-170. [CrossRef] [PubMed]

29. Saharan, V.V.; Verma, P.; Singh, A.P. High prevalence of antimicrobial resistance in Escherichia coli, Salmonella spp. and Staphylococcus aureus isolated from fish samples in India. Aquac. Res. 2019, 51, 1200-1210. [CrossRef]

30. Mok, J.S.; Cho, S.R.; Park, Y.J.; Jo, M.R.; Ha, K.S.; Kim, P.H.; Kim, M.J. Distribution and antimicrobial resistance of Vibrio parahaemolyticus isolated from fish and shrimp aquaculture farms along the Korean coast. Mar. Pollut. Bull. 2021, $171,112785$. [CrossRef] [PubMed]

31. Delalay, G.; Berezowski, J.A.; Diserens, N.; Schmidt-Posthaus, H. An understated danger: Antimicrobial resistance in aquaculture and pet fish in Switzerland, a retrospective study from 2000 to 2017. J. Fish Dis. 2020, 43, 1299-1315. [CrossRef]

32. Tyagi, A.; Singh, B.; ThammeGowda, N.K.B.; Singh, N.K. Shotgun metagenomics offers novel insights into taxonomic compositions, metabolic pathways and antibiotic resistance genes in fish gut microbiome. Arch. Microbiol. 2019, 201, 295-303. [CrossRef]

33. Ye, L.; Liu, G.; Yao, T.; Lu, J. Monitoring of antimicrobial resistance genes in the spotted sea bass (Lateolabrax maculatus): Association with the microbiome and its environment in aquaculture ponds. Environ. Pollut. 2021, 276, 116714. [CrossRef]

34. Saenz, J.S.; Marques, T.V.; Barone, R.S.C.; Cyrino, J.E.P.; Kublik, S.; Nesme, J.; Schloter, M.; Rath, S.; Vestergaard, G. Oral administration of antibiotics increased the potential mobility of bacterial resistance genes in the gut of the fish Piaractus mesopotamicus. Microbiome 2019, 8, 197. [CrossRef]

35. Shefat, S.H.T. Vaccines for Use in Finfish Aquaculture. Acta Sci. Pharm. Sci. 2018, 2, 15-19.

36. Holman, D.; Yang, W.; Alexander, T.W. Antibiotic treatment in feedlot cattle: A longitudinal study of the effect of oxytetracycline and tulathromycin on the fecal and nasopharyngeal microbiota. Microbiome 2019, 7, 1-14. [CrossRef]

37. Ghanbari, M.; Klose, V.; Crispie, F.; Cotter, P.D. The dynamics of the antibiotic resistome in the feces of freshly weaned pigs following therapeutic administration of oxytetracycline. Sci. Rep. 2019, 9, 1-11. [CrossRef] [PubMed] 
38. Abraham, T.J.; Julinta, R.B.; Roy, A.; Singha, J.; Patil, P.K.; Kumar, K.A.; Paria, P.; Behera, B.K. Dietary therapeutic dose of oxytetracycline negatively influences the antioxidant capacity and immune-related genes expression in Nile tilapia Oreochromis niloticus (L.). Environ. Toxicol. Pharmacol. 2021, 87, 103685. [CrossRef] [PubMed]

39. Limbu, S.M.; Zhang, H.; Luo, Y.; Chen, L.-Q.; Zhang, M.; Du, Z.-Y. High carbohydrate diet partially protects Nile tilapia (Oreochromis niloticus) from oxytetracycline-induced side effects. Environ. Pollut. 2019, 256, 113508. [CrossRef] [PubMed]

40. Reda, R.; Ibrahim, R.; Ahmed, E.-N.G.; El-Bouhy, Z. Effect of oxytetracycline and florfenicol as growth promoters on the health status of cultured Oreochromis niloticus. Egypt. J. Aquat. Res. 2013, 39, 241-248. [CrossRef]

41. Robinson, K.; Becker, S.; Xiao, Y.; Lyu, W.; Yang, Q.; Zhu, H.; Yang, H.; Zhao, J.; Zhang, G. Differential Impact of Subtherapeutic Antibiotics and Ionophores on Intestinal Microbiota of Broilers. Microorganisms 2019, 7, 282. [CrossRef]

42. Ramírez, C.; Coronado, J.; Silva, A.; Romero, J. Cetobacterium Is a Major Component of the Microbiome of Giant Amazonian Fish (Arapaima gigas) in Ecuador. Animals 2018, 8, 189. [CrossRef]

43. Suphoronski, S.A.; Chideroli, R.; Facimoto, C.T.; Mainardi, R.M.; de Souza, F.P.; Lopera-Barrero, N.M.; Jesus, G.F.A.; Martins, M.L.; Di Santis, G.W.; De Oliveira, A.; et al. Effects of a phytogenic, alone and associated with potassium diformate, on tilapia growth, immunity, gut microbiome and resistance against francisellosis. Sci. Rep. 2019, 9, 1-14. [CrossRef]

44. Chang, X.; Li, H.; Feng, J.; Chen, Y.; Nie, G.; Zhang, J. Effects of cadmium exposure on the composition and diversity of the intestinal microbial community of common carp (Cyprinus carpio L.). Ecotoxicol. Environ. Saf. 2018, 171, 92-98. [CrossRef]

45. Zhou, L.; Limbu, S.; Qiao, F.; Du, Z.-Y.; Zhang, M. Influence of Long-Term Feeding Antibiotics on the Gut Health of Zebrafish. Zebrafish 2018, 15, 340-348. [CrossRef] [PubMed]

46. Li, J.; Dong, T.; Keerthisinghe, T.P.; Chen, H.; Li, M.; Chu, W.; Yang, J.; Hu, Z.; Snyder, S.A.; Dong, W.; et al. Long-term oxytetracycline exposure potentially alters brain thyroid hormone and serotonin homeostasis in zebrafish. J. Hazard. Mater. 2020, 399, 123061. [CrossRef] [PubMed]

47. Gupta, S.; Fernandes, J.; Kiron, V. Antibiotic-Induced Perturbations Are Manifested in the Dominant Intestinal Bacterial Phyla of Atlantic Salmon. Microorganisms 2019, 7, 233. [CrossRef] [PubMed]

48. Smith, P.; Hiney, M.; Samuelsen, O.B. Bacterial resistance to antimicrobial agents used in fish farming: A critical evaluation of method and meaning. Annu. Rev. Fish Dis. 1994, 4, 273-313. [CrossRef]

49. Kholil, I.; Hossain, M.M.; Neowajh, S.; Islam, S.; Kabir, M. Comparative efficiency of some commercial antibiotics against Pseudomonas infection in fish. Int. J. Fish. Aquat. Stud. 2015, 2, 114-117. [CrossRef]

50. Serrano, P.H. Responsible use of antibiotics in aquaculture. In FAO Fisheries Technical Paper; FAO: Rome, Italy, 2005; Volume 469, p. 9. ISBN 9251054363.

51. Zhang, Y.; Wen, B.; David, M.A.; Gao, J.-Z.; Chen, Z.-Z. Comparative analysis of intestinal microbiota of discus fish (Symphysodon haraldi) with different growth rates. Aquaculture 2021, 540, 736740. [CrossRef]

52. Zhang, H.; Ding, Q.; Wang, A.; Liu, Y.; Teame, T.; Ran, C.; Yang, Y.; He, S.; Zhou, W.; Olsen, R.E.; et al. Effects of dietary sodium acetate on food intake, weight gain, intestinal digestive enzyme activities, energy metabolism and gut microbiota in cultured fish: Zebrafish as a model. Aquaculture 2020, 523, 735188. [CrossRef]

53. Wang, H.; Gu, Y.; Zhou, H.; Chen, J.; Wang, M.; Jiang, H.; Cao, H. Plesiomonas shigelloides, a potential pathogen of enteritis in Ictalurus punctatus. Isr. J. Aquac. Bamidgeh 2020, 72, 1-11. [CrossRef]

54. Adesiyan, I.M.; Bisi-Johnson, M.A.; Ogunfowokan, A.O.; Okoh, A. Incidence and antimicrobial susceptibility fingerprints of Plesiomonas shigelliodes isolates in water samples collected from some freshwater resources in Southwest Nigeria. Sci. Total. Environ. 2019, 665, 632-640. [CrossRef]

55. Jun, J.W.; Kim, J.H.; Jr, C.H.C.; Shin, S.P.; Han, J.E.; Jeong, D.S.; Park, S.C. Isolation and molecular detection of Plesiomonas shigelloides containing tetA gene from Asian arowana (Scleropages formosus) in a Korean aquarium. African J. Microbiol. Res. 2011, 5, 5019-5021. [CrossRef]

56. Martins, A.; Pinheiro, T.; Imperatori, A.; Freire, S.; Sá-Freire, L.; Moreira, B.; Bonelli, R. Plesiomonas shigelloides: A notable carrier of acquired antimicrobial resistance in small aquaculture farms. Aquaculture 2018, 500, 514-520. [CrossRef]

57. Grossman, T.H. Tetracycline Antibiotics and Resistance. Cold Spring Harb. Perspect. Med. 2016, 6, a025387. [CrossRef]

58. Fatahi-Bafghi, M. Antibiotic resistance genes in the Actinobacteria phylum. Eur. J. Clin. Microbiol. Infect. Dis. 2019, 38, 1599-1624. [CrossRef]

59. Wang, A.R.; Ran, C.; Ringø, E.; Zhou, Z.G. Progress in fish gastrointestinal microbiota research. Rev. Aquac. 2017, 10, 626-640. [CrossRef]

60. Egerton, S.; Culloty, S.; Whooley, J.; Stanton, C.; Ross, R. The Gut Microbiota of Marine Fish. Front. Microbiol. 2018, 9, 873. [CrossRef]

61. De Vries, L.E.; Vallès, Y.; Agersø, Y.; Vaishampayan, P.A.; García-Montaner, A.; Kuehl, J.V.; Christensen, H.; Barlow, M.; Francino, M.P. The Gut as Reservoir of Antibiotic Resistance: Microbial Diversity of Tetracycline Resistance in Mother and Infant. PLoS ONE 2011, 6, e21644. [CrossRef] [PubMed]

62. Talwar, C.; Nagar, S.; Lal, R.; Negi, R.K. Fish Gut Microbiome: Current Approaches and Future Perspectives. Indian J. Microbiol. 2018, 58, 397-414. [CrossRef] [PubMed]

63. Narrowe, A.B.; Albuthi-Lantz, M.; Smith, E.P.; Bower, K.J.; Roane, T.M.; Vajda, A.M.; Miller, C.S. Perturbation and restoration of the fathead minnow gut microbiome after low-level triclosan exposure. Microbiome 2015, 3, 1-18. [CrossRef] 
64. Fjellheim, A.J.; Playfoot, K.J.; Skjermo, J.; Vadstein, O. Inter-individual variation in the dominant intestinal microbiota of reared Atlantic cod (Gadus morhua L.) larvae. Aquac. Res. 2011, 43, 1499-1508. [CrossRef]

65. Kim, H.B.; Borewicz, K.; White, B.A.; Singer, R.S.; Sreevatsan, S.; Tu, Z.J.; Isaacson, R.E. Longitudinal investigation of the age-related bacterial diversity in the feces of commercial pigs. Veter. Microbiol. 2011, 153, 124-133. [CrossRef]

66. Stanley, D.; Geier, M.S.; Hughes, R.J.; Denman, S.; Moore, R.J. Highly Variable Microbiota Development in the Chicken Gastrointestinal Tract. PLoS ONE 2013, 8, e84290. [CrossRef]

67. Webster, T.M.U.; Consuegra, S.; Hitchings, M.; de Leaniz, C.G. Interpopulation Variation in the Atlantic Salmon Microbiome Reflects Environmental and Genetic Diversity. Appl. Environ. Microbiol. 2018, 84, e00691-18. [CrossRef]

68. Mansfield, G.S.; Desai, A.R.; Nilson, S.A.; Van Kessel, A.G.; Drew, M.D.; Hill, J.E. Characterization of rainbow trout (Oncorhynchus mykiss) intestinal microbiota and inflammatory marker gene expression in a recirculating aquaculture system. Aquaculture 2010, 307, 95-104. [CrossRef]

69. Valdés, N.; Gonzalez, A.; Garcia, V.; Tello, M. Analysis of the Microbiome of Rainbow Trout (Oncorhynchus mykiss) Exposed to the Pathogen Flavobacterium psychrophilum 10094. Microbiol. Resour. Announc. 2020, 9, e01562-19. [CrossRef]

70. Winand, R.; Bogaerts, B.; Hoffman, S.; Lefevre, L.; Delvoye, M.; Van Braekel, J.; Fu, Q.; Roosens, N.H.; De Keersmaecker, S.C.; Vanneste, K. Targeting the 16S rRNA Gene for Bacterial Identification in Complex Mixed Samples: Comparative Evaluation of Second (Illumina) and Third (Oxford Nanopore Technologies) Generation Sequencing Technologies. Int. J. Mol. Sci. 2019, 21, 298. [CrossRef] [PubMed]

71. Schurch, N.J.; Schofield, P.; Gierliński, M.; Cole, C.; Sherstnev, A.; Singh, V.; Wrobel, N.; Gharbi, K.; Simpson, G.G.; Owen-Hughes, T.; et al. How many biological replicates are needed in an RNA-seq experiment and which differential expression tool should you use? RNA 2016, 22, 839-851. [CrossRef] [PubMed]

72. Bara, M.; Joffe, A.R. The ethical dimension in published animal research in critical care: The public face of science. Crit. Care 2014, 18, R15. [CrossRef] [PubMed]

73. Lyons, P.P.; Turnbull, J.; Dawson, K.A.; Crumlish, M. Exploring the microbial diversity of the distal intestinal lumen and mucosa of farmed rainbow troutOncorhynchus mykiss(Walbaum) using next generation sequencing (NGS). Aquac. Res. 2015, 48, 77-91. [CrossRef]

74. Knudsen, B.E.; Bergmark, L.; Munk, P.; Lukjancenko, O.; Priemé, A.; Aarestrup, F.; Pamp, S.J. Impact of Sample Type and DNA Isolation Procedure on Genomic Inference of Microbiome Composition. mSystems 2016, 1, e00095-16. [CrossRef]

75. Huang, W.; Cheng, Z.; Lei, S.; Liu, L.; Lv, X.; Chen, L.; Wu, M.; Wang, C.; Tian, B.; Song, Y. Community composition, diversity, and metabolism of intestinal microbiota in cultivated European eel (Anguilla anguilla). Appl. Microbiol. Biotechnol. 2018, 102, $4143-4157$. [CrossRef]

76. Kiruthiga, R.; Thanislass, J.; Antony, P.X.; Lydia, D.C.; Uma-Maheswari, D.; Venkatesa-Perumal, S. Analysis of V3 Region of 16S rRNA for the Characterization of Rumen Microbiome of Goat. J. Anim. Sci. Res. 2018, 2. [CrossRef]

77. Ma, L.; Xie, Y.; Han, Z.; Giesy, J.P.; Zhang, X. Responses of earthworms and microbial communities in their guts to Triclosan. Chemosphere 2016, 168, 1194-1202. [CrossRef] [PubMed]

78. Huang, L.; Xu, Y.-B.; Xu, J.-X.; Ling, J.-Y.; Chen, J.-L.; Zhou, J.-L.; Zheng, L.; Du, Q.-P. Antibiotic resistance genes (ARGs) in duck and fish production ponds with integrated or non-integrated mode. Chemosphere 2017, 168, 1107-1114. [CrossRef] [PubMed]

79. Schloss, P.D.; Westcott, S.L.; Ryabin, T.; Hall, J.R.; Hartmann, M.; Hollister, E.B.; Lesniewski, R.A.; Oakley, B.B.; Parks, D.H.; Robinson, C.J.; et al. Introducing mothur: Open-Source, Platform-Independent, Community-Supported Software for Describing and Comparing Microbial Communities. Appl. Environ. Microbiol. 2009, 75, 7537-7541. [CrossRef] [PubMed]

80. Edgar, R.C.; Haas, B.J.; Clemente, J.C.; Quince, C.; Knight, R. UCHIME improves sensitivity and speed of chimera detection. Bioinformatics 2011, 27, 2194-2200. [CrossRef]

81. Quast, C.; Pruesse, E.; Yilmaz, P.; Gerken, J.; Schweer, T.; Yarza, P.; Peplies, J.; Glöckner, F.O. The SILVA ribosomal RNA gene database project: Improved data processing and web-based tools. Nucleic Acids Res. 2012, 41, D590-D596. [CrossRef]

82. Wickham, H. ggplot2. Wiley Interdiscip. Rev. Comput. Stat. 2011, 3, 180-185. [CrossRef]

83. McMurdie, P.; Holmes, S. phyloseq: An R Package for Reproducible Interactive Analysis and Graphics of Microbiome Census Data. PLoS ONE 2013, 8, e61217. [CrossRef]

84. Wickham, H. Reshaping Data with thereshapePackage. J. Stat. Softw. 2007, 21, 1-20. [CrossRef]

85. Oksanen, J. vegan: Community Ecology Package, R Package Version 1.8-5; 2007. Available online: https:/ /CRAN.R-project.org/ package=vegan (accessed on 20 May 2019).

86. Yue, J.C.; Clayton, M.K. A Similarity Measure Based on Species Proportions. Commun. Stat. Theory Methods 2005, 34, $2123-2131$. [CrossRef]

87. Bray, J.R.; Curtis, J.T. An Ordination of the Upland Forest Communities of Southern Wisconsin. Ecol. Monogr. 1957, 27, 325-349. [CrossRef]

88. Anderson, M.J. A new method for non-parametric multivariate analysis of variance. Austral Ecol. 2001, 26, 32-46. [CrossRef]

89. Fox, J.; Muenchen, R.; Putler, D. Package 'RcmdrMisc'; 2018. Available online: https://CRAN.R-project.org/package=RcmdrMisc (accessed on 1 April 2020). 